\title{
ON WEYL MODULES OVER AFFINE LIE ALGEBRAS IN PRIME CHARACTERISTIC
}

\author{
CHUN-JU LAI
}

\begin{abstract}
We construct a family of homomorphisms between Weyl modules for affine Lie algebras in characteristic $p$, which supports our conjecture on the strong linkage principle in this context. We also exhibit a large class of reducible Weyl modules beyond level one, for $p$ not necessarily small.
\end{abstract}

\section{INTRODUCTION}

1.1. The theory of modular representations of reductive algebraic groups is well established [Jan03]. In this context, the Weyl modules play a fundamental role as the Verma modules for the category $\mathcal{O}$, as Lusztig's conjecture provides a precise formula expressing the irreducible character in terms of characters of Weyl modules, where the highest weights of these Weyl modules are controlled by the so-called strong linkage principle - in other words, if a simple module is a composition factor of a Weyl module then the two highest weights are "linked" together - this notion is introduced by Andersen [And80], following the notion of "linkage principle" given by Humphreys [Hum71.

Explicit homomorphisms between Weyl modules have been constructed by CarterLusztig [CL74] and by Carter-Payne [CP80] for type $A$. For arbitrary types, homomorphisms have been constructed by Franklin [Fra81, Fra88. Franklin's work is based on Shapovalov's construction [Sha72] on homomorphisms between Verma modules in characteristic 0. He first constructed an integral version of Shapovalov elements, which gives rise to homomorphisms between the $\mathbb{Z}$-forms of Verma modules. By modifying integral Shapovalov elements, one defines a map between the Weyl modules using reduction modulo $p$. On the one hand, a detailed calculation involving the contravariant form is needed to make sure the map is nonzero. On the other hand, the BGG resolution is needed to ensure that the map is indeed a homomorphism.

We are interested in developing a theory of modular representations of affine Lie algebras. So far there are very few results in the literature. For one, Mathieu Mat96 showed that the Steinberg modules are not irreducible in contrast with the finite types. For another, DeConcini-Kac-Kazhdan [DKK89] showed that the basic representations for untwisted affine ADE types are irreducible if and only if the characteristic $p$ of the underlying field does not divide the determinant of the Cartan matrix of the underlying finite root system. Chari-Jing [CJ01] further showed that the level one representations are irreducible if $p$ is coprime to the Coxeter number $h$. For (possibly twisted) affine ADE types, Brundan-Kleshchev 
BK02 computed explicitly the determinant of the Shapovalov form on weight spaces of the basic representations. When combined with [DKK89], it implies that the basic representation is irreducible if $p>3$ for (possibly twisted) affine DE types and if $p>h$ for (possibly twisted) affine A types. The success of all these works above essentially hinges on concrete realizations of basic representations of affine Lie algebras via vertex operators. In contrast, little is known about the irreducibility of Weyl modules beyond level one.

A major difficulty in generalizing modular representation theory of finite types is that in an affine Weyl group there are no longest elements, which play an important role in the finite types. For example, the strong linkage principle for modular finite types can be proved either by the Jantzen sum formula [Jan77] under some assumption on $p$ or by Andersen's approach And80] on the cohomology on line bundles. In either way, the proof highly relies on the existence of the longest element of a Weyl group.

The main observation of this article is that Franklin's method can be generalized to the affine case as long as one establishes the affine version of the integral Shapovalov elements. Here we state our main theorem.

Main Theorem. Assume that $\lambda, \mu$ are dominant integral weights such that $\mu=$ $\lambda-D \gamma$ for some positive real root $\gamma$ and positive integer $D$. There exists a nonzero homomorphism $V(\mu) \rightarrow V(\lambda)$ between Weyl modules of affine Lie algebras in prime characteristic if the following conditions hold.

(1) $\mu$ is $\gamma$-mirrored to $\lambda$ (cf. \$2.7).

(2) $\gamma$ is "good" (cf. \$4.1).

(3) D is "small enough" (cf. Theorem 5.10).

In this article we also formulate a conjecture (Conjecture 6.1) on the strong linkage principle for Weyl modules over affine Lie algebras. To support our conjecture, we construct a large class of reducible Weyl modules beyond level one using the main theorem, for $p$ not necessarily small. In particular, our results indicate that any clear formulation of a sufficient condition for the irreducibility of Weyl modules seems to be too subtle to formulate.

For type $\widetilde{A}_{r}$, we also give an upper bound for $p$ in terms of the level such that the main theorem applies. In particular, for type $\widetilde{A}_{1}$ and for the first few primes we give a table showing the lowest level of reducible Weyl modules that arise from our main theorem, and the complete list of quasi-simple weights (cf. $\lcm{6.3}$ ) of level less than 150 .

1.2. The paper is organized as follows. In Section 2 we introduce notations that are needed for the rest of this paper.

In Section 3 we construct the integral Shapovalov elements for affine Lie algebras. These elements, by definition, give rise to nonzero homomorphisms between Verma modules for affine Lie algebras over $\mathbb{Z}$ if the two highest weights are linked by a reflection in the affine Weyl group. 
In Section 4 we show that if the two weights are $\gamma$-mirrored for some positive real root $\gamma$, the integral Shapovalov elements actually give rise to nonzero homomorphisms between Verma modules in the characteristic $p$, under a condition on the distance between the two highest weights. We then show that condition can be weakened by modifying the integral Shapovalov elements, provided $\gamma$ is "good".

In Section 5 we show that if two $\gamma$-mirrored weights satisfy an additional condition on their distance, an analogous nonzero homomorphisms between the corresponding Weyl modules can be constructed. The contravariant forms and the BGG resolution for Kac-Moody algebras are used in the proof. Another essential tool here is Lemma 5.2, which is a variant of the Shapovalov determinant formula.

In Section 6 we relate our main theorem to our conjecture on the strong linkage principle. We also describe the candidates for reducible Weyl modules. Using our main theorem, we show that most candidates are reducible except for some rare "quasi-simple weights". For type $\widetilde{A}_{1}$ we computed for the first few primes a list of reducible Weyl modules that can be detected by our main Theorem; for type $\widetilde{A}_{r}$ we also give an upper bound for $p$ in terms of the level such that our main theorem applies.

Acknowledgments. I am grateful to my advisor, Professor Weiqiang Wang, for his guidance and support. I would like to thank Professor Leonard Scott for insightful discussions and bringing Franklin's paper to our attention. I must thank Professor James Franklin for providing his unpublished thesis paper. I thank Professor Toshiyuki Tanisaki for pointing out an ambiguity in the paper. I would also like to thank Professor Jim Humphreys and Shrawan Kumar for useful comments.

This research is partially supported by the GRA fellowship of Wang's NSF grant DMS-1101268. We thank Institute of Mathematics, Academia Sinica, Taipei for providing an excellent working environment and support, where part of this project was carried out.

\section{Preliminaries}

2.1. Affine Kac-Moody Algebras. Let $\mathfrak{g}_{\mathbb{C}}=\mathfrak{g}_{\mathbb{C}}(A)$ be the affine Kac-Moody algebra over $\mathbb{C}$ associated to a symmetrizable generalized Cartan matrix (GCM) $A=\left(a_{i j}\right)_{i, j \in I}$ of affine type and of rank $r$ where $I=\{0,1, \ldots, r\}$. Following Kac90] and Car05], let $\vec{a}=\left(a_{i}\right)_{i \in I}$ and $\vec{c}=\left(c_{i}\right)_{i \in I}$ be the minimal positive integer vectors such that $A \vec{a}=0=\vec{c}^{t} A$. Let $\left(\mathfrak{h}_{\mathbb{C}},\left(\alpha_{i}\right)_{i \in I},\left(h_{i}\right)_{i \in I}\right)$ be a minimal realization. Here $\mathfrak{h}_{\mathbb{C}}$ is the $\mathbb{C}$-span of $h_{0}, \ldots, h_{r}$, and $d \in \mathfrak{h}_{\mathbb{C}}$ is a fixed scaling element such that $\alpha_{i}(d)=\delta_{i, 0}$. Let $\mathfrak{n}_{\mathbb{C}}^{+}$(and $\mathfrak{n}_{\mathbb{C}}^{-}$) be the subalgebra of $\mathfrak{g}_{\mathbb{C}}$ generated by $\left(e_{i}\right)_{i \in I}$ (and $\left(f_{i}\right)_{i \in I}$, respectively). Let $\mathfrak{b}_{\mathbb{C}}^{ \pm}=\mathfrak{h}_{\mathbb{C}} \oplus \mathfrak{n}_{\mathbb{C}}^{ \pm}$be the corresponding Borel subalgebras. Let $():, \mathfrak{g}_{\mathbb{C}} \times \mathfrak{g}_{\mathbb{C}} \rightarrow \mathbb{C}$ be a non-degenerate invariant symmetric bilinear form which induces a non-degenerate bilinear form on $\mathfrak{h}_{\mathbb{C}}^{*}$.

Let $\delta=\sum_{i \in I} a_{i} \alpha_{i}$ be the basic imaginary root, let $c=\sum_{i \in I} c_{i} h_{i}$ be the central element, and fix a vector $\rho \in \mathfrak{h}_{\mathbb{C}}^{*}$ satisfying $\rho\left(h_{i}\right)=1$ for all $i \in I$. Let $h=\sum_{i \in I} a_{i}$ be the Coxeter number, and let $h^{\vee}=\sum_{i \in I} c_{i}$ be the dual Coxeter number. Let $X=\left\{\lambda \in \mathfrak{h}_{\mathbb{C}}^{*} \mid \lambda\left(h_{i}\right) \in \mathbb{Z}\right.$ for all $\left.i \in I\right\}$ be the set of integral weights, and let 
$X^{+}=\left\{\lambda \in X \mid \lambda\left(h_{i}\right) \geq 0\right.$ for all $\left.i \in I\right\}$ be the set of dominant integral weights. Let $\varpi_{0}, \ldots, \varpi_{r} \in \mathfrak{h}_{\mathbb{C}}^{*}$ be the fundamental weights, that is, each $\varpi_{j}$ is an element in $\mathfrak{h}_{\mathbb{C}}^{*}$ such that $\varpi_{j}\left(h_{i}\right)=\delta_{i, j}$ and $\varpi_{j}(d)=0$. Therefore

$$
X^{+}=\left\{\lambda=\sum_{i=0}^{r} \xi_{i} \varpi_{i}+\xi \delta \in \mathfrak{h}_{\mathbb{C}}^{*} \mid \xi_{i} \in \mathbb{Z}_{\geq 0} \text { for all } i \in I, \xi \in \mathbb{C}\right\} .
$$

2.2. Affine root systems. Let $\Phi, \Phi^{+}, \Phi_{\mathrm{re}}, \Phi_{\mathrm{re}}^{+}, \Phi_{\mathrm{im}}$ and $\Phi_{\mathrm{im}}^{+}$be the set of roots, positive roots, real roots, positive real roots, imaginary roots and positive imaginary roots of the affine Kac-Moody algebra $\mathfrak{g}_{\mathbb{C}}$, respectively. Let $\Phi_{0}$ be the root system generated by $\left\{\alpha_{i}\right\}_{i=1}^{r}$. Let $Q=\sum_{i \in I} \mathbb{Z} \alpha_{i}$ (and $Q^{+}=\sum_{i \in I} \mathbb{Z}_{\geq 0} \alpha_{i}$ ) be the set of (non-negative) integer linear combinations of roots of $\mathfrak{g}_{\mathbb{C}}$. For each element $\gamma=\sum_{i \in I} g_{i} \alpha_{i} \in Q$ for some $g_{i} \in \mathbb{Z}$, we set

$$
h_{\gamma}=\sum_{i} g_{i}^{\vee} h_{i}, \quad \text { where } g_{i}^{\vee}=\left(a_{i} / c_{i}\right) g_{i},
$$

and let ht $\gamma=\sum_{i \in I} g_{i}$ be the height of $\gamma$. A simple root $\alpha_{\eta}$ is said to be occurring in $\gamma$ if $g_{\eta} \neq 0$.

Let $\mathfrak{h}_{\mathbb{R}}$ be the $\mathbb{R}$-span of $\left\{h_{i}\right\}_{i \in I}$ and $d$. To each real root $\gamma \in \Phi_{\text {re }}$ we assign a reflection $s_{\gamma}: \mathfrak{h}_{\mathbb{R}}^{*} \rightarrow \mathfrak{h}_{\mathbb{R}}^{*}$ given by

$$
s_{\gamma}(\lambda)=\lambda-\left\langle\lambda, \gamma^{\vee}\right\rangle \gamma \text { where }\left\langle\lambda, \gamma^{\vee}\right\rangle=\frac{2(\lambda, \gamma)}{(\gamma, \gamma)} .
$$

In addition, for each $m \in \mathbb{Z}$ we assign an affine reflection $s_{\gamma, m}: \mathfrak{h}_{\mathbb{R}}^{*} \rightarrow \mathfrak{h}_{\mathbb{R}}^{*}$ given by

$$
s_{\gamma, m}(\lambda)=s_{\gamma}(\lambda)+m \gamma .
$$

The affine reflection $s_{\gamma, m}$ is the reflection with respect to the hyperplane

$$
H_{\gamma, m}=\left\{\lambda \in \mathfrak{h}_{\mathbb{R}}^{*} \mid\left\langle\lambda+\rho, \gamma^{\vee}\right\rangle=m\right\} .
$$

2.3. Affine Weyl groups. Let $W$ be the Weyl group of $\mathfrak{g}_{\mathbb{C}}$ generated by $s_{\alpha}$ with $\alpha \in \Phi_{\text {re. }}$. It is known that $W$ is also generated by the affine reflections $s_{\alpha, m}$ with $\alpha \in \Phi_{0}, m \in \mathbb{Z}$. We introduce the dot action of $W$ on $\mathfrak{h}_{\mathbb{R}}^{*}$ by

$$
w \cdot \lambda=w(\lambda+\rho)-\rho .
$$

In particular, for each real root $\alpha$ we have

$$
s_{\alpha, m} \cdot \lambda=\lambda-\left(\left\langle\lambda+\rho, \alpha^{\vee}\right\rangle-m\right) \alpha .
$$

Let $\theta$ be the highest root in $\Phi_{0}$. Then $(W, \mathcal{S})$ is a Coxeter system where the generating set $\mathcal{S}=\left\{s_{\theta, 1}, s_{\alpha_{1}}, \ldots, s_{\alpha_{r}}\right\}$. A reduced expression for $w \in W$ is a product $w=t_{1} \cdots t_{N}$ of elements $\left\{t_{i}\right\}_{i=1}^{N}$ in $\mathcal{S}$ such that the number $N$ is minimal among all such expressions for $w$. Here $l(w)=N$ is called the length of $w$. 
2.4. Verma modules. For any Lie algebra $\mathfrak{a}$ let $U(\mathfrak{a})$ be its universal enveloping algebra. Let $U=U\left(\mathfrak{g}_{\mathbb{C}}\right)$, and let $\mathfrak{h}_{\mathbb{Z}}$ be the $\mathbb{Z}$-span of $\left\{d, h_{i} \mid i \in I\right\}$. Denote the $\mathbb{Z}$-form of $U$ by

$$
U_{\mathbb{Z}}=\left\langle e_{i}^{(n)}, f_{i}^{(n)},\left(\begin{array}{l}
h \\
n
\end{array}\right) \mid i \in I, n \in \mathbb{Z}_{>0}, h \in \mathfrak{h}_{\mathbb{Z}}\right\rangle,
$$

where $e_{i}^{(n)}=e_{i}^{n} / n !, f_{i}^{(n)}=f_{i}^{n} / n$ ! and $\left(\begin{array}{l}h \\ n\end{array}\right)=h(h-1) \cdots(h-n+1) / n !$. We denote by $U_{\mathbb{Z}}^{-}$and $U_{\mathbb{Z}}^{+}$the subrings of $U$ generated by $f_{i}^{(n)}$ and by $e_{i}^{(n)}$, respectively. Let $\mathfrak{g}_{\mathbb{Z}}=\mathfrak{g}_{\mathbb{C}} \cap U_{\mathbb{Z}}, \mathfrak{n}_{\mathbb{Z}}^{ \pm}=\mathfrak{n}_{\mathbb{C}}^{ \pm} \cap U_{\mathbb{Z}}^{ \pm}$and $\mathfrak{b}_{\mathbb{Z}}^{ \pm}=\mathfrak{n}_{\mathbb{Z}}^{ \pm} \oplus \mathfrak{h}_{\mathbb{Z}}$

Let $\mathbb{K}$ be an algebraically closed field, let $\mathfrak{g}_{\mathbb{K}}, \mathfrak{n}_{\mathbb{K}}^{ \pm}, \mathfrak{h}_{\mathbb{K}}, U_{\mathbb{K}}$ and $U_{\mathbb{K}}^{ \pm}$be the tensor product of $\mathbb{K}$ with $\mathfrak{g}_{\mathbb{Z}}, \mathfrak{n}_{\mathbb{Z}}^{ \pm}, \mathfrak{h}_{\mathbb{Z}}, U_{\mathbb{Z}}$ and $U_{\mathbb{Z}}^{ \pm}$, respectively. For each $\lambda \in \mathfrak{h}_{\mathbb{Z}}^{*}$ we denote the Verma module (as a $U_{\mathbb{K}}$-module) by

$$
M(\lambda)_{\mathbb{K}}=U_{\mathbb{K}} / I(\lambda)_{\mathbb{K}},
$$

where $I(\lambda)_{\mathbb{K}}=I(\lambda)_{\mathbb{Z}} \otimes \mathbb{K}$ and

$$
I(\lambda)_{\mathbb{Z}}=\sum_{j \in I, m \geq 1} U_{\mathbb{Z}} e_{j}^{(m)}+\sum_{j \in I, m \geq 1} U\left(\mathfrak{b}_{\mathbb{Z}}^{-}\right)\left(\begin{array}{c}
h_{j}-\lambda\left(h_{j}\right) \\
m
\end{array}\right) .
$$

Let $v_{\lambda, \mathbb{K}}^{+}=1+I(\lambda)_{\mathbb{K}}$ be a highest weight vector of $M(\lambda)_{\mathbb{K}}$ and let $v_{\lambda}^{+}=v_{\lambda, \mathbb{C}}^{+}$. The Verma module $M(\lambda)_{\mathbb{C}}$ contains a unique maximal submodule $N(\lambda)_{\mathbb{C}}$ and a unique simple quotient $L(\lambda)_{\mathbb{C}}=M(\lambda)_{\mathbb{C}} / N(\lambda)_{\mathbb{C}}$.

In [KK79, Theorem 2] Kac and Kazhdan proved the strong linkage principle for symmetrisable Kac-Moody algebras.

Proposition 2.1. $L(\mu)_{\mathbb{C}}$ is a composition factor of $M(\lambda)_{\mathbb{C}}$ if and only if $\mu=\lambda$ or $\mu=\mu_{0} \uparrow \cdots \uparrow \mu_{N}=\lambda$ where

$$
x \uparrow y \Leftrightarrow \text { there exists }\left\{\begin{array} { l } 
{ n \in \mathbb { Z } _ { > 0 } , } \\
{ \beta \in \Phi ^ { + } }
\end{array} \text { such that } \left\{\begin{array}{l}
y-x=n \beta, \\
n(\beta, \beta)=2(y+\rho, \beta) .
\end{array}\right.\right.
$$

Remark 2.2. The notion of "linkage principle" - all composition factors of an indecomposable have linked highest weights - is introduced by Humphreys in [Hum71]. The notion of the strong linkage principle - all composition factors have highest weights strongly linked to a certain weight - was then defined by Andersen And80, though Jantzen had earlier worked out some of the ideas in his own notation.

2.5. Integral bases for $U_{\mathbb{Z}}$. Based on Garland's work Gar78 on the loop algebras, Mitzman constructed a PBW-type basis for all (possibly twisted) affine Lie algebras. Here we first recall the root vectors defined in [Mit85] define such a basis for each choice of root vector; then fix a choice (cf. Proposition 2.4) that significantly simplifies the computation.

Assume that $\mathfrak{g}_{\mathbb{C}}$ is of type $X_{r}^{(k)}$. Let $\sigma$ be the Dynkin diagram automorphism of order $k$ which fixes vertex 0 . For $1 \leq i \leq r$ and $n \neq 0$, we set

$$
X_{n \delta, i}= \begin{cases}\frac{1}{2} X_{n \delta, \alpha_{i}} & \text { if }\left(\alpha_{i}, \sigma\left(\alpha_{i}\right)\right) \text { is odd and } n \text { is even, } \\ X_{n \delta, \alpha_{i}} & \text { otherwise. }\end{cases}
$$


Here $X_{n \delta, \alpha_{i}}$ is the vector defined in [Mit85, (3.9.1), (3.9.6)] (was denoted by $X_{n \imath, \mathrm{a}}$ ). We then choose $X_{\gamma}$ for $\gamma \in \Phi_{\text {re }}^{+}$such that the set $\left\{X_{\gamma} \mid \gamma \in \Phi_{\text {re }}^{+}\right\} \cup\left\{X_{n \delta, i} \mid 1 \leq\right.$ $i \leq r, n \neq 0\} \cup\left\{h_{0}, h_{1}, \ldots, h_{r}, d\right\}$ is a Chevally basis of $\mathfrak{g}_{\mathbb{C}}$ in the sense of [Mit85, Definition 2.2.25].

Fix a total ordering $\leq$ on the set $\Phi_{M}^{+}=\left\{(\gamma, i) \in \Phi^{+} \times \mathbb{Z} \mid 1 \leq i \leq \operatorname{dim} \mathfrak{g}_{\mathbb{C}, \gamma}\right\}$. For now we fix root vectors

$$
\left\{f_{\gamma, i}, e_{\gamma, i} \mid(\gamma, i) \in \Phi_{M}^{+}\right\},
$$

where $e_{\gamma, 1}=X_{\gamma}, f_{\gamma, 1}=X_{-\gamma}$ if $\gamma \in \Phi_{\mathrm{re}}^{+} ; e_{\gamma, i}=X_{\gamma, i}, f_{\gamma, i}=X_{-\gamma, i}$ if $\gamma \in \Phi_{\mathrm{im}}^{+}$. For short, we write $f_{\gamma}=f_{\gamma, 1}$ and $e_{\gamma}=e_{\gamma, 1}$ for $\gamma \in \Phi_{\text {re }}^{+}$; write $e_{i}=e_{\alpha_{i}}$ and $f_{i}=f_{\alpha_{i}}$ for $i \in I$. Define polynomials $\Lambda_{s}=\Lambda_{s}\left(x_{1}, x_{2}, x_{3}, \ldots\right)$ over $\mathbb{C}\left[x_{1}, x_{2}, x_{3}, \ldots\right][[\zeta]]$ for $s \geq 0$ by

$$
\sum_{s \geq 0} \Lambda_{s} \zeta^{s}=\exp \left(\sum_{j \geq 1} x_{j} \frac{\zeta^{j}}{j}\right)
$$

For $(\gamma, i) \in \Phi_{M}^{+}, h \in \mathfrak{h}_{\mathbb{Z}}, s \geq 0$, by a slight abuse of notation, set

$$
f_{\gamma, i}^{(s)}=\Lambda_{s}\left(f_{\gamma, i}, f_{2 \gamma, i}, f_{3 \gamma, i}, \ldots\right), e_{\gamma, i}^{(s)}=\Lambda_{s}\left(e_{\gamma, i}, e_{2 \gamma, i}, e_{3 \gamma, i}, \ldots\right) \text {, and } h^{(s)}=\Lambda_{s}(h, h, \ldots) .
$$

Note that by [Mit85, $(4.2 .9-10)], f_{\gamma, 1}^{(s)}=f_{\gamma}^{s} / s$ ! and $e_{\gamma, 1}^{(s)}=e_{\gamma}^{s} / s$ ! are the usual divided powers if $\gamma \in \Phi_{\text {re }}^{+}$, and $h^{(s)}=\left(\begin{array}{c}h+s-1 \\ s\end{array}\right)$ for $h \in \mathfrak{h}_{\mathbb{Z}}$. Let $\mathcal{P}$ be the set of functions mapping from $\Phi_{M}^{+}$to $\mathbb{Z}_{\geq 0}$ with finite support. For each $\pi \in \mathcal{P}$ with finite support $\left\{\left(\gamma_{1}, i_{1}\right)<\cdots<\left(\gamma_{m}, i_{m}\right)\right\}$ we denote the degree of $\pi$ by $\operatorname{deg} \pi=$ $\sum_{j=1}^{m} \pi\left(\gamma_{j}, i_{j}\right) \in \mathbb{Z}_{\geq 0}$, the weight of $\pi$ by $\operatorname{wt}(\pi)=\sum_{j=1}^{N} \pi\left(\gamma_{j}, i_{j}\right) \gamma_{j} \in Q^{+}$. We define

$$
\begin{aligned}
f_{\pi} & =f_{\left.\left.\gamma_{1}, i_{1}, i_{1}\right)\right)}^{\left(\pi \left(\gamma_{1}, i_{1}\right.\right.} \cdots f_{\left.\left.\gamma_{m}, i_{m}, i_{m}\right)\right)}^{\left(\pi\left(\gamma_{m}, i_{m}\right)\right.} \in U_{\mathbb{Z}}^{-}, \text {and } \\
e_{\pi} & =e_{\gamma_{1}, i_{1}}^{\left(\pi\left(\gamma_{1}, i_{1}\right)\right)} \cdots e_{\gamma_{m}, i_{m}}^{\left(\pi\left(\gamma_{m}, i_{m}\right)\right)} \in U_{\mathbb{Z}}^{+} .
\end{aligned}
$$

Similarly, for each tuple $\varphi=\left(\varphi_{0}, \varphi_{1}, \ldots \varphi_{r}, \varphi_{r+1}\right) \in \mathbb{Z}_{\geq 0}^{r+2}$ we assign

$$
h_{\varphi}=h_{0}^{\left(\varphi_{0}\right)} \cdots h_{r}^{\left(\varphi_{r}\right)} d^{\left(\varphi_{r+1}\right)} \in U\left(\mathfrak{h}_{\mathbb{Z}}\right) .
$$

Lemma 2.3. (Mitzman) $U_{\mathbb{Z}}$ has a Garland-Kostant-type $\mathbb{Z}$-basis

$$
\left\{f_{\pi} h_{\varphi} e_{\pi^{\prime}} \mid \pi, \pi^{\prime} \in \mathcal{P}, \varphi \in \mathbb{Z}_{\geq 0}^{r+2}\right\} .
$$

As a consequence, $U$ has a PBW-type $\mathbb{C}$-basis

$$
\left\{F_{\pi} H_{\varphi} E_{\pi^{\prime}} \mid \pi, \pi^{\prime} \in \mathcal{P}, \varphi \in \mathbb{Z}_{\geq 0}^{r+2}\right\}
$$

where

$$
F_{\pi}=f_{\pi} \prod_{j=1}^{m} \pi\left(\gamma_{j}, i_{j}\right) !, \quad E_{\pi}=e_{\pi} \prod_{j=1}^{m} \pi\left(\gamma_{j}, i_{j}\right) !, \quad H_{\varphi}=h_{\varphi} \prod_{j=0}^{r+1} \varphi_{j} !
$$

Proof. By [Mit85, Theorem 4.2.6], the set of monomials $f_{\pi} h_{\varphi} e_{\pi^{\prime}}$ forms a $\mathbb{Z}$-basis of the subalgebra of $U$ generated by $f_{\gamma, i}^{(s)}, e_{\gamma, i}^{(s)}, h_{j}^{(s)}$ and $d^{(s)}$ for $(\gamma, i) \in \Phi_{M}^{+}, 0 \leq j \leq$ $r, s \geq 0$. See [Mit85, Remark 4.2.7, Corollary 4.4.12] for the identification of this subalgebra with the algebra $U_{\mathbb{Z}}$ defined in Section 2.4. 
We are able to express each $u \in U\left(\mathfrak{b}_{\mathbb{C}}^{-}\right)$as

$$
u=\sum_{\pi \in \mathcal{P}} F_{\pi} Q_{\pi},
$$

where $Q_{\pi} \in U\left(\mathfrak{h}_{\mathbb{C}}\right) \simeq \mathbb{C}\left[h_{0}, \ldots, h_{r}, d\right]$. We further denote the evaluation of $u$ at $\lambda \in \mathfrak{h}_{\mathbb{C}}^{*}$ by

$$
u(\lambda)=\sum_{\pi \in \mathcal{P}} F_{\pi} Q_{\pi}(\lambda) \in U\left(\mathfrak{n}_{\mathbb{C}}^{-}\right)
$$

Now we explain how we pick a choice of the root vectors and hence an integral basis of $U_{\mathbb{Z}}$. For $\omega \in \mathcal{P}, q \in \mathbb{Z}_{>0}$ and a simple root $\alpha$, we may rewrite $f_{\alpha}^{q} F_{\omega}$, using relations in $U$, as a $\mathbb{C}$-linear combination of monomials of the form

$$
F_{\pi} f_{\alpha}^{q-i}, \text { where } \pi(\alpha, 1)=0, i \in \mathbb{Z} .
$$

That is, we "move" $f_{\alpha}$ to the very right end. This operation shows up frequently when it comes to solving the Shapovalov-type equations in Section 3. Now we define $C_{\omega, \pi, i, q} \in \mathbb{C}$ be the coefficients such that

$$
f_{\alpha}^{m} F_{\omega}=\sum_{\substack{\pi \in \mathcal{P} \\ \pi(\alpha, 1)=0}} \sum_{i \in \mathbb{Z}} F_{\pi} f_{\alpha}^{m-i} C_{\omega, \pi, i, m}
$$

Proposition 2.4. We can rescale the root vectors (cf. (2.4)) so that there are polynomials $C_{\omega, \pi, i} \in \mathbb{Z}[m]$ satisfying $C_{\omega, \pi, i, m}=C_{\omega, \pi, i}(m)$ for all $\omega, \pi, i$ and $m$.

Proof. The proof is exactly the same as in the finite case (see Fra88, Lemma $4.1])$.

From now on, by a little abuse of notation, fix $\left\{f_{\gamma, i}, e_{\gamma, i}\right\}$ to be a set of root vectors as in Proposition 2.4, and hence we have

$$
f_{\alpha}^{m} F_{\omega}=\sum_{\substack{\pi \in \mathcal{P} \\ \pi(\alpha)=0}} \sum_{i \in \mathbb{Z}} F_{\pi} f_{\alpha}^{m-i} C_{\omega, \pi, i}(m)
$$

for each $m \in \mathbb{Z}_{>0}, \omega \in \mathcal{P}$ and simple root $\alpha$.

Example 2.5. Consider the affine Kac-Moody algebra of type $\widetilde{A}_{1}$ with GCM

$$
A=\left[\begin{array}{cc}
2 & -2 \\
-2 & 2
\end{array}\right]
$$

Let $\gamma=\alpha_{0}+\delta$ and pick root vectors $f_{0}, f_{1}, f_{\delta, 1}$ and $f_{\gamma}$ satisfying

$$
f_{\delta, 1}=f_{0} f_{1}-f_{1} f_{0} \text { and } f_{\gamma}=f_{0} f_{\delta, 1}-f_{\delta, 1} f_{0} .
$$

Fixing $\alpha=\alpha_{0}$ and $F_{\omega}=f_{0}^{2} f_{1}=f_{1} f_{0}^{2}+2 f_{\delta, 1} f_{0}+f_{\gamma}$, we have

$$
f_{0}^{m} F_{\omega}=\left(f_{1} f_{0}^{2}+(m+2) f_{\delta, 1} f_{0}+\left(\begin{array}{c}
m+2 \\
2
\end{array}\right) f_{\gamma}\right) f_{0}^{m}
$$


that is,

$$
C_{\omega, \pi, i}(m)= \begin{cases}1 & \text { if } F_{\pi}=f_{1}, i=-2, \\
m+2 & \text { if } F_{\pi}=f_{\delta, 1}, i=-1, \\
\left(\begin{array}{c}
m+2 \\
2
\end{array}\right) & \text { if } F_{\pi}=f_{\gamma}, i=0, \\
0 & \text { otherwise. }\end{cases}
$$

Note that $\left(\begin{array}{c}m+2 \\ 2\end{array}\right) \notin \mathbb{Z}[m]$, so we have to choose again $f_{\gamma}=\frac{1}{2}\left(f_{0} f_{\delta, 1}-f_{\delta, 1} f_{0}\right)$ to make $C_{\omega, \pi, i}(m) \in \mathbb{Z}[m]$ as mentioned in Proposition [2.4.

2.6. Weyl modules. Note that in the finite dimensional framework there are two constructions of the Weyl modules - one by reduction modulo $p$ from known modules in characteristic 0 , and one by taking dual of some induced modules. In this paper we adopt the definition of Weyl modules by reduction modulo $p$. From now on let $\mathbb{K}$ be an algebraically closed field of characteristic $p>0$. For each $\lambda \in X^{+}$, recall that $M(\lambda)_{\mathbb{C}}=U v_{\lambda}^{+}$so that we have

$$
L(\lambda)_{\mathbb{C}}=U \bar{v}_{\lambda}^{+} \text {where } \bar{v}_{\lambda}^{+}=v_{\lambda}^{+}+N(\lambda)_{\mathbb{C}}
$$

Then we define its $\mathbb{Z}$-form $L(\lambda)_{\mathbb{Z}}=U_{\mathbb{Z}} \bar{v}_{\lambda}^{+}$and define the Weyl module

$$
V(\lambda)=L(\lambda)_{\mathbb{Z}} \otimes \mathbb{K}
$$

2.7. Nearest lower reflections. Fix a positive real root $\gamma \in \Phi_{\text {re }}^{+}$and a positive integer $e$, for each $\lambda \in X$ we set $D^{\prime}=\left\langle\lambda+\rho, \gamma^{\vee}\right\rangle \in \mathbb{Z}$. There are uniquely determined integers $M, D \in \mathbb{Z}$ such that $D^{\prime}=M p^{e}+D$ such that $0 \leq D<p^{e}$. Set $R_{\gamma, e}=s_{\gamma, M p^{e}}$, hence

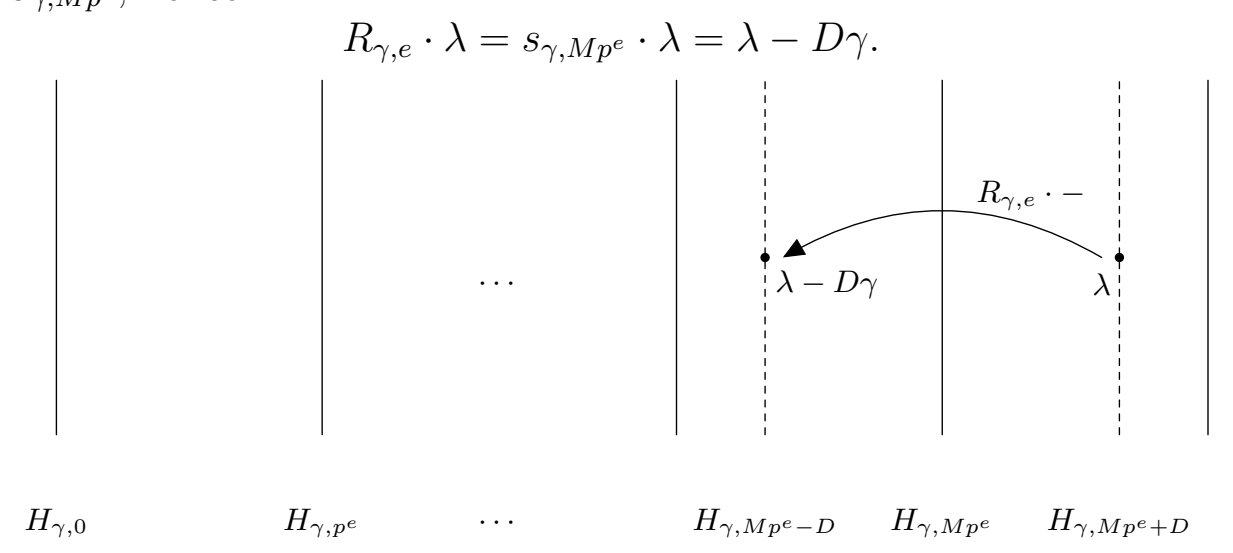

In words, $R_{\gamma, e}$ is the nearest lower $p^{e}$-reflection. That is, among those $p^{e}$-hyperplanes of the form $H_{\gamma, M p^{e}}$ with $M \in \mathbb{Z}, R_{\gamma, e}$ is the reflection with respect to the hyperplane that is nearest to $\lambda$.

Let $\lambda, \mu \in X^{+}$be two dominant integral weights. $\mu$ is said to be $(\gamma, e)$-mirrored to $\lambda$ if $\mu=R_{\gamma, e} \cdot \lambda$ and we write $\mu \uparrow_{\gamma}^{e} \lambda$. Finally, $\mu$ is said to be $\gamma$-mirrored to $\lambda$ if $\mu$ is $(\gamma, e)$-mirrored to $\lambda$ for some positive integer $e$ and we write $\mu \uparrow_{\gamma} \lambda$.

Remark 2.6. In modular finite case, the blocks were classified by Donkin [Don80] using the theory of $G_{n} T$-modules. Here $G_{n}$ is the $n$th Frobenius kernel in the algebraic group $G$ and $T$ is a maximal torus in $G$. In the representation theory for 
$G_{n} T$, the building blocks of strong linkage are given by the $\gamma$-mirrored condition. The strong linkage principle in this context is proved by Doty Dot89] and he showed that it implies the strong linkage principle for the modular finite case. This is why our seemingly strange $\gamma$-mirrored condition is the reasonable format.

\section{Construction of Integral Shapovalov elements}

In this section we construct an integral version of Shapovalov elements for the affine case. The argument is adapted from Franklin's construction [Fra88 for the finite case. The idea is to study closely the coefficients in every Shapovalov equation in terms of the PBW-type basis for affine Lie algebras.

3.1. Generic Shapovalov Elements. As in [KK79], it is easy to verify that if $\chi \in H_{\gamma, D}$ then any nonzero homomorphism $M(\chi-D \gamma)_{\mathbb{C}} \rightarrow M(\chi)_{\mathbb{C}}$ is an embedding and is of the form $v_{\chi-D \gamma}^{+} \mapsto u v_{\chi}^{+}$for some $u \in U\left(\mathfrak{b}_{\mathbb{C}}^{-}\right)_{-D \gamma}$. On the other hand, for a fixed positive real root $\gamma$ and a fixed positive integer $D$, any generic Shapovalov element $S$ (with respect to $(\gamma, D)$ ) gives rise to nonzero homomorphisms $M(\chi-$ $D \gamma)_{\mathbb{C}} \rightarrow M(\chi)_{\mathbb{C}}$ by $v_{\chi-D \gamma}^{+} \mapsto S v_{\chi}^{+}$if $\chi \in H_{\gamma, D}$.

Definition 3.1. An element $S \in U\left(\mathfrak{b}_{\mathbb{C}}^{-}\right)$is called a (generic) Shapovalov element (with respect to a positive real root $\gamma$ and a positive integer $D$ ) if the conditions (S1)-(S3) below hold:

$(\mathrm{S} 1) S \in U\left(\mathfrak{b}_{\mathbb{C}}^{-}\right)_{-D \gamma}$;

(S2) $e_{i} S \cdot v_{\chi}^{+}=0$ for all $i \in I$ and $\chi \in H_{\gamma, D}$;

(S3) The highest degree PBW monomial of $S$ is a product of $f_{i}^{D g_{i}}$ for all $i \in I$.

Lemma 3.2. Let $\gamma \in \Phi_{r e}^{+}$and $D \in \mathbb{Z}_{>0}$. There is a Zariski-dense subset $\Theta$ of the hyperplane $H_{\gamma, D}$ such that for each $\chi \in \Theta$ there exists an element $S^{\chi}=S^{\chi}(\gamma, D)$ in $U\left(\mathfrak{n}_{\mathbb{C}}^{-}\right)$satisfying $\left(S 1^{\prime}\right)-\left(S 3^{\prime}\right)$ below:

$\left(\mathrm{S}^{\prime}\right) S^{\chi}=\sum_{\pi \in \mathcal{P}} F_{\pi} Q_{\pi}^{\chi}$ where $w t(\pi)=D \gamma$ and $Q_{\pi}^{\chi} \in \mathbb{C} ;$

$\left(\mathrm{S} 2^{\prime}\right) S^{\chi} \cdot v_{\chi}^{+}$is a singular vector in $M(\chi)_{\mathbb{C}}$;

$\left(\mathrm{S}^{\prime}\right)$ The highest degree PBW monomial of $S^{\chi}$ is a product of $f_{i}^{D g_{i}}$ for all $i \in I$. Moreover, $\Theta$ satisfies a polynomial condition below:

$\left(\mathrm{S}^{\prime}\right)$ There are polynomials $Q_{\pi} \in \mathbb{C}\left[h_{0}, \ldots, h_{r}\right]$ such that $Q_{\pi}(\chi)=Q_{\pi}^{\chi}$ for $\chi \in \Theta$.

Proof. The proof is exactly the same as in the finite case (see [Hum08, Sect. 4.13]).

Corollary 3.3. For fixed $\gamma \in \Phi_{r e}^{+}$and $D \in \mathbb{Z}_{>0}$, there exists a (generic) Shapovalov element $S=S(\gamma, D)$. Moreover, $S$ is unique modulo the left $U\left(\mathfrak{b}_{\mathbb{C}}^{-}\right)$-ideal $J(\gamma, D)=$ $U\left(\mathfrak{b}_{\mathbb{C}}^{-}\right)\left(h_{\gamma}+\rho\left(h_{\gamma}\right)-D\right)$.

Proof. Let $S=\sum_{\pi} F_{\pi} Q_{\pi}$ where the polynomials $Q_{\pi}$ are defined in Lemma 3.2, For each $\chi \in \Theta$ the evaluation $S(\chi)$ equals to the element $S^{\chi}$ constructed in Lemma 3.2. By the density of $\Theta$, the evaluation $S(\chi)$ also satisfies Conditions $\left(\mathrm{S}^{\prime}\right)-\left(\mathrm{S} 3^{\prime}\right)$ for each $\chi \in H_{\gamma, D}$ and hence $S$ satisfies Conditions (S1) - (S3). 
3.2. Integral Shapovalov Elements. The above-mentioned generic Shapovalov elements give rise to nonzero homomorphisms between Verma modules in characteristic 0. Franklin ([Fra81]) introduced the integral Shapovalov elements for finite types and showed that such elements give rise to nonzero homomorphisms between the $\mathbb{Z}$-forms of Verma modules for finite types.

We generalize his idea to the affine types and showed that the integral Shapovalov elements give rise to nonzero homomorphisms between the $\mathbb{Z}$-forms of Verma modules for affine types. In this section we mainly follow the outline given in [Fra88, while some adaptations are needed for the affine type.

Definition 3.4. An element $Z \in U\left(\mathfrak{b}_{\mathbb{C}}^{-}\right)$is called an integral Shapovalov element (with respect to a positive real root $\gamma=\sum_{i \in I} g_{i} \alpha_{i}, g_{i} \in \mathbb{Z}$; and a positive integer $D)$ if the conditions (Z1)-(Z3) below hold:

(Z1) $Z=\sum_{\pi \in \mathcal{P}} F_{\pi} Q_{\pi}$ where $\operatorname{wt}(\pi)=D \gamma$ and $Q_{\pi} \in \mathbb{Z}\left[h_{0}, \ldots, h_{r}\right]$;

$(\mathrm{Z} 2) e_{i}^{(n)} Z \in I(\gamma, D)_{\mathbb{Z}}=\sum_{\substack{j \in I \\ m \geq 1}} U_{\mathbb{Z}} e_{j}^{(m)}+U\left(\mathfrak{b}_{\mathbb{Z}}^{-}\right)\left(h_{\gamma}+\rho\left(h_{\gamma}\right)-D\right)$ for $i \in I, n>0 ;$

(Z3) The highest degree PBW monomial in $Z$ is a product of $f_{i}^{D g_{i}}$ for all $i \in I$.

We will see that for each $\gamma \in \Phi_{\mathrm{re}}^{+}$and $D \in \mathbb{Z}_{>0}$, the integral Shapovalov element does exist and is unique (cf. 3.6), which we call $Z(\gamma, D)$. Here we prove the existence of $Z(\gamma, D)$ and its "evaluation" $Z^{\chi}(\gamma, D)$ simultaneously via an induction on the height of $\gamma$ as follows:

(1) The initial case follows from an explicit construction.

(2) Assuming the existence of $Z(\beta, D)$ for some $\beta=s_{\alpha}(\gamma)<\gamma$, we construct explicitly a dense subset $\Theta_{1}=\Theta_{1}(\alpha)$ of $H_{\gamma, D}$ (cf. (3.2) ) such that for any $\chi \in \Theta_{1}, Z^{\chi}$ exists.

(3) The existence of $Z^{\chi}$ in a dense subset $\Theta_{1}$ of $H_{\gamma, D}$ leads to the existence of $Z(\gamma, D)$.

Theorem 3.5. Let $\gamma=\sum_{i \in I} g_{i} \alpha_{i} \in \Phi_{r e}^{+}$and $D \in \mathbb{Z}_{>0}$. There is a dense subset $\Theta \subset H_{\gamma, D}$ such that for each $\chi \in \Theta$ there exists $Z^{\chi}=Z^{\chi}(\gamma, D) \in U\left(\mathfrak{n}_{\mathbb{C}}^{-}\right)$satisfying $\left(Z 1^{\prime}\right)-\left(Z 3^{\prime}\right)$ below:

$\left(\mathrm{Z} 1^{\prime}\right) Z^{\chi}=\sum_{\pi \in \mathcal{P}} F_{\pi} Q_{\pi}^{\chi}$ where $w t(\pi)=D \gamma$ and $Q_{\pi}^{\chi} \in \mathbb{Z}$

$\left(\mathrm{Z} 2^{\prime}\right) e_{i}^{(n)} Z^{\chi} \in I(\chi)_{\mathbb{Z}}(c f .(2.2))$ for $i \in I, n \in \mathbb{Z}_{>0}$;

$\left(\mathrm{Z} 3^{\prime}\right)$ The highest degree $P B W$ monomial in $Z^{\chi}$ is a product of $f_{i}^{D g_{i}}$ for all $i \in I$;

$\left(\mathrm{Z} 4^{\prime}\right)$ There are polynomials $Q_{\pi} \in \mathbb{Z}\left[h_{0}, \ldots, h_{r}\right]$ such that $Q_{\pi}(\chi)=Q_{\pi}^{\chi}$ for $\chi \in \Theta$. Assuming Theorem 3.5, one can prove the existence and uniqueness immediately.

Corollary 3.6. For each $\gamma \in \Phi_{r e}^{+}$and $D \in \mathbb{Z}_{>0}$ there exists an integral Shapovalov element $Z=Z(\gamma, D) \in U\left(\mathfrak{b}_{\mathbb{Z}}^{-}\right)$. Moreover, $Z$ is unique modulo the left $U\left(\mathfrak{b}_{\mathbb{Z}}^{-}\right)$-ideal

$$
J(\gamma, D)_{\mathbb{Z}}=U\left(\mathfrak{b}_{\mathbb{Z}}^{-}\right)\left(h_{\gamma}+\rho\left(h_{\gamma}\right)-D\right) .
$$

Proof. The existence part is similar to Corollary 3.3. The uniqueness part follows from that $e_{i}^{(n)} J(\gamma, D)_{\mathbb{Z}} \subset U\left(\mathfrak{b}_{\mathbb{Z}}^{-}\right) e_{i}^{(n)}+U\left(\mathfrak{b}_{\mathbb{Z}}^{-}\right)\left(h_{\gamma}+\rho\left(h_{\gamma}\right)-D\right) \subset I(\gamma, D)_{\mathbb{Z}}$. 
Before proceeding to the proof of Theorem 3.5 we need some lemmas.

Lemma 3.7. If $\gamma \in \Phi_{r e}^{+}$then there are distinct positive roots $\left\{\gamma_{i}\right\}_{i=0}^{n}$ such that

(1) $\gamma_{0}=\gamma$.

(2) $\gamma_{i}=s_{\epsilon_{i}}\left(\gamma_{i-1}\right)<\gamma_{i-1}$ for some simple root $\epsilon_{i}$.

(3) $\gamma_{n}=\alpha_{\eta}$ for some simple root $\alpha_{\eta}$ occurring (cf. S2.2) in $\gamma$.

Proof. This is done by induction on the height of $\gamma$.

Remark 3.8. For any $\lambda \in H_{\gamma, M p^{e}+D}$, we have a commutative diagram of weights via dot-actions:

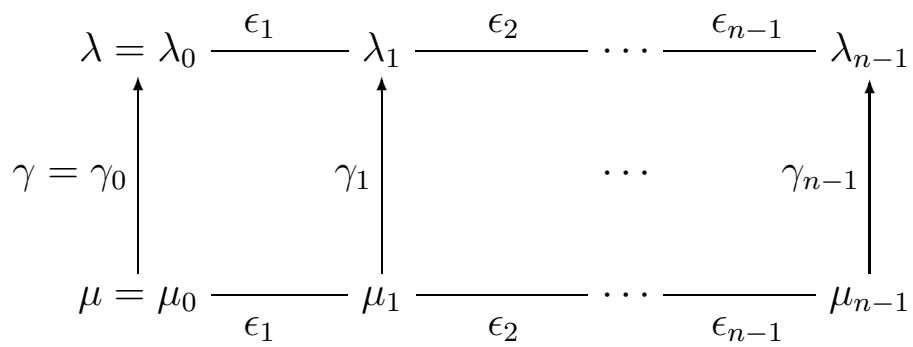

where $\lambda_{i}=s_{\epsilon_{i}} \cdot \lambda_{i-1}, \mu_{i}=s_{\epsilon_{i}} \cdot \mu_{i-1}=\lambda_{i}-D \gamma$, and $\mu=\lambda-D \gamma$.

Note that we reserve the freedom to choose a suitable dense subset of $H_{\gamma, M p^{e}+D}$ to determine the order of the $\lambda_{i}$ 's and $\mu_{i}$ 's, and hence the direction of the corresponding Verma module inclusions.

In the rest of this section we fix $\gamma \in \Phi_{\mathrm{re}}^{+}, D \in \mathbb{Z}_{>0}, \chi \in H_{\gamma, D} \cap X, \nu=s_{\alpha} \cdot \chi$ and $\beta=s_{\alpha}(\gamma)<\gamma$ for a simple root $\alpha$. We have

$$
\begin{aligned}
& \nu=\chi+q \alpha \text { where } q=-\left\langle\chi+\rho, \alpha^{\vee}\right\rangle \in \mathbb{Z}, \text { and } \\
& \beta=\gamma-b \alpha \text { where } b=\left\langle\gamma, \alpha^{\vee}\right\rangle \in \mathbb{Z}_{>0} .
\end{aligned}
$$

There are three possible commutative diagrams of weights via dot-actions:

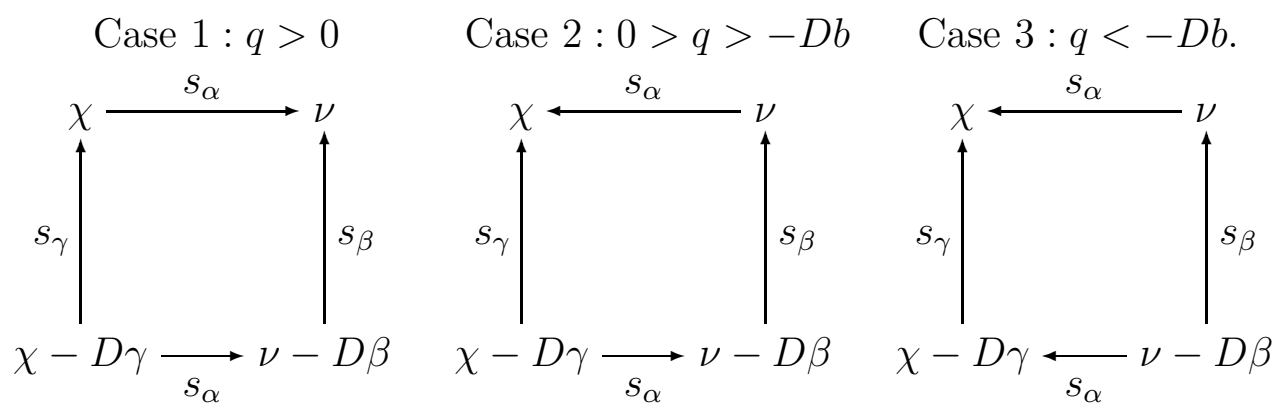

The first case will be used in proving Theorem 3.5, the second case shows up in the proof of Lemma 5.2, although one can formulate a similar statement for the third case, it does not play a role in this article. Let

$$
\Theta_{1}=\Theta_{1}(\gamma, D, \alpha)=H_{\gamma, D} \cap X \cap H_{\alpha, 0}^{-},
$$

where $H_{\alpha, m}^{-}=\left\{\chi \in \mathfrak{h}_{\mathbb{R}}^{*} \mid\left\langle\chi+\rho, \alpha^{\vee}\right\rangle<m\right\}$. In other words, we have $q, q+D b \in \mathbb{Z}_{>0}$ for each $\chi \in \Theta_{1}$. We further let

$$
\Theta_{2}=\Theta_{2}(\gamma, D, \alpha)=H_{\gamma, D} \cap X \cap H_{\alpha, 0}^{+} \cap H_{\alpha,-D b}^{-},
$$


where $H_{\alpha, m}^{+}=\left\{\chi \in \mathfrak{h}_{\mathbb{R}}^{*} \mid\left\langle\chi+\rho, \alpha^{\vee}\right\rangle>m\right\}$ for all $m \in \mathbb{Z}$. In contrast, we have $q \in \mathbb{Z}_{<0}$ and $q+D b \in \mathbb{Z}_{>0}$ for each $\chi \in \Theta_{2}$.

Lemma 3.9. Assume that $Z=Z(\beta, D)$ exists, then each $\chi \in \Theta_{1}$ (cf. (3.2)) has a unique (depending only on the choice of $Z$ ) element $Z^{\chi}=Z^{\chi}(\gamma, D, \alpha) \in U\left(\mathfrak{n}_{\mathbb{Z}}^{-}\right)_{-D \gamma}$ making the following diagram of Verma module homomorphisms commute.

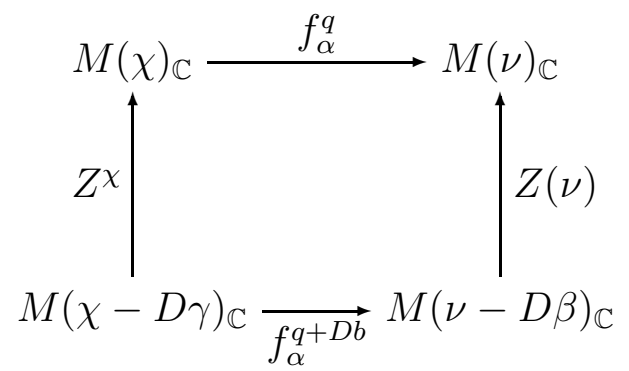

Here $Z(\nu)$ is the evaluation of $Z$ at $\nu$ (cf. (2.5). In particular, the element $Z^{\chi}$ is determined by the Shapovalov equation

$$
Z^{\chi} f_{\alpha}^{q}=f_{\alpha}^{q+D b} Z(\nu)
$$

Proof. A direct calculation shows that $\nu-D \beta=s_{\beta} \cdot \nu=s_{\alpha} \cdot(\chi-D \gamma)$, and hence by the strong linkage principle (Proposition 2.1) we have a commuting diagram of nonzero homomorphisms below.

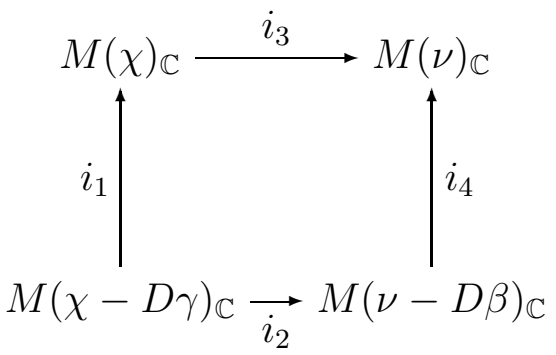

Here $i_{k}$ is the left multiplication of a nonzero element $u_{k} \in U\left(\mathfrak{n}_{\mathbb{C}}^{-}\right)$for $k=1, \ldots, 4$. Since $f_{\alpha}^{q} \in \mathbb{C} u_{3}, f_{\alpha}^{q+D b} \in \mathbb{C} u_{2}$ and by assumption that $Z(\nu) \in \mathbb{C} u_{4}$, so there is a unique element $Z^{\chi} \in \mathbb{C} u_{1}$ such that the Shapovalov equation holds.

Similarly, one can prove the counterpart of Lemma 3.9.

Lemma 3.10. Assume that $Z=Z(\beta, D)$ exists, then each $\chi \in \Theta_{2}$ (cf. (3.3) ) has a unique (depending only on the choice of $Z$ ) element $Z^{\chi}=Z^{\chi}(\gamma, D, \alpha) \in U\left(\mathfrak{n}_{\mathbb{Z}}^{-}\right)_{-D \gamma}$ such that the following diagram of Verma module homomorphisms commutes.

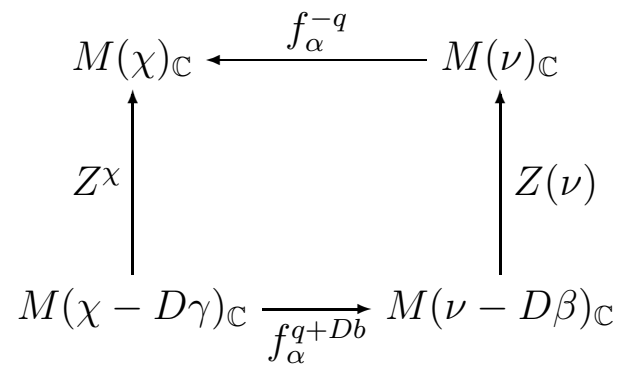


In particular, the element $Z^{\chi}$ is determined by the Shapovalov equation

$$
Z^{\chi}=f_{\alpha}^{q+D b} Z(\nu) f_{\alpha}^{-q}
$$

Now we are finally in a position to prove Theorem 3.5 .

Proof of Theorem 3.5. The proof is done by induction on the height of $\gamma$. For the initial case $\gamma=\alpha_{\eta}$, we set $\Theta=H_{\gamma, D} \cap X$ and $Z^{\chi}=f_{\eta}^{D}$ so that the conditions $\left(\mathrm{Z1}^{\prime}\right)$, $\left(\mathrm{Z}^{\prime}\right)$ and $\left(\mathrm{Z}^{\prime}\right)$ follow immediately from definition. Finally $\left(\mathrm{Z}^{\prime}\right)$ follows from the useful formula of Kostant below.

$$
e_{i}^{(n)} f_{\eta}^{(D)}=\delta_{i, \eta} \sum_{k=0}^{\min (n, D)} f_{i}^{(D-k)}\left(\begin{array}{c}
h_{i}-n-D+2 k \\
k
\end{array}\right) e_{i}^{(n-k)} .
$$

For the inductive case ht $\gamma>1$, we apply Lemma 3.9 with $\alpha=\epsilon_{1}, \Theta=\Theta_{1}$ and obtain a Shapovalov equation $Z^{\chi} f_{\alpha}^{q}=f_{\alpha}^{q+D b} Z(\nu)$. Let $Z(\nu)=\sum_{\omega} F_{\omega} Q_{\omega}^{\prime}$ where $Q_{\omega}^{\prime} \in \mathbb{Z}$. Solving this leads to an explicit expression:

$$
\begin{aligned}
Z^{\chi} f_{\alpha}^{q} & =\sum_{\omega \in \mathcal{P}} f_{\alpha}^{q+D b} F_{\omega} Q_{\omega}^{\prime} \\
& =\sum_{\substack{\pi \in \mathcal{P} \\
\pi(\alpha)=0}} \sum_{i \in \mathbb{Z}} F_{\pi} f_{\alpha}^{q+D b-i}\left(\sum_{\omega \in \mathcal{P}} C_{\omega, \pi, i}(q+D b) Q_{\omega}^{\prime}\right) .
\end{aligned}
$$

Therefore, $Z^{\chi}=\sum_{\pi} F_{\pi} Q_{\pi}^{\chi}$ where

$$
Q_{\pi}^{\chi}=\sum_{\omega \in \mathcal{P}} C_{\omega} Q_{\omega}^{\prime} \text { for some } C_{\omega} \in \mathbb{Z}
$$

It is routine to check that the element $Z^{\chi}$ obtained this way satisfies $\left(\mathrm{Z}^{\prime}\right)-\left(\mathrm{Z3^{ \prime }}\right)$. For $\left(\mathrm{Z}^{\prime}\right)$, by the inductive hypothesis for each $\pi$ there is a polynomial $P_{\pi} \in$ $\mathbb{Z}\left[h_{0}, \ldots, h_{r}\right]$ such that $Q_{\pi}^{\prime}=P_{\pi}(\nu)$. Let $\alpha=\alpha_{k}$. Note that for each $i \in I$ we have

$$
h_{i}(\nu)=\left\langle\nu, \alpha_{i}^{\vee}\right\rangle=\left\langle\chi, \alpha_{i}^{\vee}\right\rangle-\left\langle\chi+\rho, \alpha^{\vee}\right\rangle\left\langle\alpha, \alpha_{i}^{\vee}\right\rangle=\left(h_{i}-a_{i k}\left(h_{k}+1\right)\right)(\chi) .
$$

So there is a polynomial $P_{\pi}^{\prime} \in \mathbb{Z}\left[h_{0}, \ldots, h_{r}\right]$, obtained from $P_{\pi}$ by replacing $h_{i}$ by $h_{i}-a_{i k}\left(h_{k}+1\right)$ for each $i$, such that $P_{\pi}^{\prime}(\chi)=P_{\pi}(\nu)=Q_{\pi}^{\prime}$ and hence by defining $Q_{\pi}=\sum_{\omega \in \mathcal{P}} C_{\omega} P_{\pi}^{\prime}$ one has

$$
Q_{\pi}(\chi)=\sum_{\omega \in \mathcal{P}} C_{\omega} P_{\pi}^{\prime}(\chi)=\sum_{\omega \in \mathcal{P}} C_{\omega} Q_{\pi}^{\prime}=Q_{\pi}^{\chi}
$$

Corollary 3.11. Assume that $\lambda \in H_{\gamma, D}$ and $\mu=\lambda-D \gamma$. There is a nonzero homomorphism $M(\mu)_{\mathbb{Z}} \rightarrow M(\lambda)_{\mathbb{Z}}$ given by

$$
v_{\mu}^{+} \mapsto Z v_{\lambda}^{+},
$$

where $Z=Z(\gamma, D)$ is the integral Shapovalov element. 
Example 3.12. Consider the affine Kac-Moody algebra of type $\widetilde{A}_{1}$ with fixed positive real root $\gamma=\alpha_{0}+\delta$ and $D=1$. Then the sequence of positive roots described in Lemma 3.7 is given by

$$
\alpha_{0}+\delta=\gamma_{0}>\gamma_{1}=s_{\alpha_{0}}\left(\gamma_{0}\right)=\alpha_{1}
$$

The dense subset $\Theta \subset H_{\gamma, D}$ in Theorem 3.5 is given by

$$
\begin{aligned}
\Theta & =\left\{\chi=\xi_{0} \varpi_{0}-2\left(\xi_{0}+1\right) \varpi_{1}+\xi \delta \mid \xi_{0} \in \mathbb{Z}_{<0}\right\} \\
& =\left\{-(q+1) \varpi_{0}+2 q \varpi_{1}+\xi \delta \mid q \in \mathbb{Z}_{\geq 0}\right\},
\end{aligned}
$$

where $q=-\left\langle\chi+\rho, \alpha_{0}^{\vee}\right\rangle$. For each weight $\chi \in \Theta$, we have a Shapovalov equation

$$
Z^{\chi} f_{0}^{q}=f_{0}^{q+2} f_{1}
$$

Solving this, we get $Z^{\chi}=f_{1} f_{0}^{2}+f_{\delta, 1} f_{0}(q+2)+f_{\gamma}(q+2)(q+1)$ as in Example 2.5. Note that evaluation at $\chi=-(q+1) \varpi_{0}+2 q \varpi_{1}+\xi \delta$ replaces $h_{0}$ by $-(q+1)$, equivalently, $q+2=-h_{0}(\chi)+1$. Hence it leads to an integral Shapovalov element

$$
Z=f_{1} f_{0}^{2}-f_{\delta, 1} f_{0}\left(h_{0}-1\right)+f_{\gamma}\left(h_{0}^{2}-h_{0}\right) \text {. }
$$

\section{Homomorphisms betWeen Verma modules in Characteristic $p$}

For finite types, Franklin ([Fra88] ) showed that if the corresponding positive root for an integral Shapovalov element is not "exceptional" , there is a "modified" Shapovalov element that gives rise to a nonzero homomorphism between Verma modules in characteristic $p$, without any restriction. As for the five "exceptional" roots, additional restrictions on the distance between the two highest weights are required.

In this section we generalize Franklin's idea to affine types. In our viewpoint, the restriction for "exceptional" roots is in fact a generic phenomenon. We first show that under a restriction on the distance, integral Shapovalov elements give rise to nonzero homomorphisms between Verma modules in characteristic $p$. We then show that the restriction is the coarsest if the corresponding positive real root is "good".

\section{1. $\eta$-goodness.}

Definition 4.1. For a fixed prime number $p$ and $\eta \in I$, a positive real root $\gamma \in \Phi_{r e}^{+}$ is called $\eta$-good if the multiplicity of $h_{\eta}$ occurring in $h_{\gamma}$ (cf. (2.1) ) is coprime to $p$, and the simple root $\alpha_{\eta}$ has the same length as $\gamma$.

In other words, assume that $h_{\gamma}=\sum g_{i}^{\vee} h_{i}$ for some $g_{i}^{\vee} \in \mathbb{Z}$. $\gamma$ is $\eta$-good if $\operatorname{gcd}\left(g_{\eta}^{\vee}, p\right)=1$ and $\left(\alpha_{\eta}, \alpha_{\eta}\right)=(\gamma, \gamma)$.

Lemma 4.2. If $\gamma$ is $\eta$-good then

$$
\mathbb{Z}\left[h_{0}, \ldots, h_{r}\right] \otimes \mathbb{Z}_{(p)} \simeq \mathbb{Z}\left[h_{0}, \ldots, \widehat{h}_{\eta}, \ldots, h_{r}, h_{\gamma}+\rho\left(h_{\gamma}\right)-D\right] \otimes \mathbb{Z}_{(p)} .
$$

Here $\widehat{h}_{\eta}$ represents the omission of the variable and $\mathbb{Z}_{(p)}=\{r / q \in \mathbb{Q} \mid \operatorname{gcd}(p, q)=$ 1) is the localization of $\mathbb{Z}$ at $p \mathbb{Z}$.

Proof. It follows from that $h_{\eta}=\frac{1}{g_{\eta}^{\vee}}\left(h_{\gamma}-\sum_{i \neq \eta} g_{i}^{\vee} h_{i}\right)$ and $p \nmid g_{\eta}^{\vee}$. 
Now we define an " $h_{\eta}$-avoiding" Shapovalov-type element by modifying $Z$ as follows.

Lemma 4.3. Let $Z=Z(\gamma, D)=\sum_{\pi} F_{\pi} Q_{\pi}$ with $Q_{\pi} \in \mathbb{Z}\left[h_{0}, \ldots, h_{r}\right]$ for some $D \in$ $\mathbb{Z}_{>0}$ and $\gamma \in \Phi_{r e}^{+}$such that $\alpha_{\eta}$ is occurring in $\gamma$, and let $N=\max \left\{\operatorname{deg}_{h_{\eta}} Q_{\pi} \mid \pi \in\right.$ $\mathcal{P}\}$. There is an element $Z_{\eta}=Z_{\eta}(\gamma, D) \in U\left(\mathfrak{b}_{\mathbb{Z}}^{-}\right)$satisfying the following conditions (G1)-(G3):

(G1) $Z_{\eta}=\sum_{\pi \in \mathcal{P}} F_{\pi} Q_{\eta, \pi}$ where $w t(\pi)=D \gamma$ and $Q_{\eta, \pi} \in \mathbb{Z}\left[h_{0}, \ldots, \widehat{h}_{\eta}, \ldots, h_{r}\right]$;

(G2) $e_{i}^{(n)} Z_{\eta} \in I(\gamma, D)_{\mathbb{Z}}$ for all $i \in I$ and $n>0$;

(G3) The highest degree $P B W$ monomial in $Z_{\eta}$ is a product of $f_{i}^{D g_{i}}$ for all $i \in I$ times $\left(g_{\eta}^{\vee}\right)^{N}$.

Proof. By definition,

$$
\left(g_{\eta}^{\vee}\right)^{N} Z=\sum_{\pi \in \mathcal{P}} F_{\pi}\left(g_{\eta}^{\vee}\right)^{N} Q_{\pi}=\sum_{\pi \in \mathcal{P}} F_{\pi} Q_{\pi}^{\prime},
$$

where $Q_{\pi}^{\prime} \in \mathbb{Z}\left[h_{0}, \ldots, \widehat{h}_{\eta}, \ldots, h_{r}, h_{\gamma}+\rho\left(h_{\gamma}\right)-D\right]$. Equivalently,

$$
\left(g_{\eta}^{\vee}\right)^{N} Z=\sum_{m=0}^{N} u_{\eta, m}\left(h_{\gamma}+\rho\left(h_{\gamma}\right)-D\right)^{m},
$$

for some $u_{\eta, m} \in U\left(\mathfrak{b}_{\mathbb{Z}}^{-}\right)$. Finally, we define

$$
Z_{\eta}=\left(g_{\eta}^{\vee}\right)^{N} Z-\sum_{m=1}^{N} u_{\eta, m}\left(h_{\gamma}+\rho\left(h_{\gamma}\right)-D\right)^{m}=\sum_{\pi \in \mathcal{P}} F_{\pi} Q_{\eta, \pi},
$$

where $Q_{\eta, \pi} \in \mathbb{Z}\left[h_{0}, \ldots, \widehat{h}_{\eta}, \ldots, h_{r}\right]$ as required in (G1). By construction (G2) is satisfied. (G3) follows from the fact that $e_{i}^{(n)}\left(h_{\gamma}+\rho\left(h_{\gamma}\right)-D\right) \in U_{\mathbb{Z}} e_{i}^{(n)} \subset I(\gamma, D)_{\mathbb{Z}}$ and hence

$$
e_{i}^{(n)} Z_{\eta}=\left(g_{\eta}^{\vee}\right)^{N} e_{i}^{(n)} Z-\sum_{m=1}^{N} u_{\eta, m} e_{i}^{(n)}\left(h_{\gamma}+\rho\left(h_{\gamma}\right)-D\right)^{m} \in I(\gamma, D)_{\mathbb{Z}} .
$$

Example 4.4. Recall that in Example 3.12 we have $\gamma=2 \alpha_{0}+\alpha_{1}$ and hence $h_{\gamma}=$ $2 h_{0}+h_{1}$. Therefore $\gamma$ is 0 -good for any odd prime and is 1-good for any prime. We have already an $h_{1}$-avoiding Shapovalov element

$$
Z_{1}=Z=f_{1} f_{0}^{2}-f_{\delta, 1} f_{0}\left(h_{0}-1\right)+f_{\gamma}\left(h_{0}^{2}-h_{0}\right) .
$$

As for $\eta=0$, to achieve an $h_{0}$-avoiding Shapovalov element we first replace $h_{0}$ by $\frac{1}{2}\left(h_{\gamma}-h_{1}\right)$ and then make a suitable scaling. Thus

$$
g_{0}^{N} Z=2^{2} Z=4 f_{1} f_{0}^{2}-2 f_{\delta, 1} f_{0}\left(h_{\gamma}-h_{1}-2\right)+f_{\gamma}\left(h_{\gamma}-h_{1}\right)\left(h_{\gamma}-h_{1}-2\right) .
$$

Note that $h_{\gamma}+\rho\left(h_{\gamma}\right)-D=h_{\gamma}+2$. Setting $h_{\gamma}=-2$, we obtain

$$
Z_{0}=4 f_{1} f_{0}^{2}+2 f_{\delta, 1} f_{0}\left(h_{1}+4\right)+f_{\gamma}\left(h_{1}+2\right)\left(h_{1}+4\right) .
$$


4.2. Projection onto $U\left(\mathfrak{b}_{\mathbb{C}}^{-}\right)$. Let $P_{\mathfrak{b}^{-}}: U \rightarrow U\left(\mathfrak{b}_{\mathbb{C}}^{-}\right)$be the projection corresponding to the direct sum decomposition $U=\sum_{\alpha \in \Phi^{+}} U e_{\alpha} \oplus U\left(\mathfrak{b}_{\mathbb{C}}^{-}\right)$. It is easy to see that for each $\lambda \in \mathfrak{h}_{\mathbb{R}}^{*}, u \in U$ we have

$$
u v_{\lambda}^{+}=P_{\mathfrak{b}^{-}}(u) v_{\lambda}^{+} .
$$

In this section we study $e_{i}^{(n)} Z_{\eta} v_{\lambda}^{+}$for the $h_{\eta}$-avoiding integral Shapovalov element $Z_{\eta}$ we constructed in Lemma 4.3.

\section{Lemma 4.5.}

(1) Assume that $n \in \mathbb{Z}_{>0}, i \in I$. Let $\Psi_{\pi, \omega}=\Psi_{\pi, \omega}(i, n) \in U\left(\mathfrak{h}_{\mathbb{C}}\right)$ be such that

$$
P_{\mathfrak{b}^{-}}\left(e_{i}^{(n)} F_{\pi}\right)=\sum_{\omega \in \mathcal{P}} F_{\omega} \Psi_{\pi, \omega} .
$$

Then $\Psi_{\pi, \omega} \in \mathbb{Z}\left[h_{i}\right]$ and $\operatorname{deg}_{h_{i}} \Psi_{\pi, \omega} \leq \pi\left(\alpha_{i}\right)$.

(2) Let $D \in \mathbb{Z}_{>0}, \gamma=\sum_{i \in I} g_{i} \alpha_{i} \in \Phi_{r e}^{+}$with $g_{\eta} \neq 0, Z_{\eta}=Z_{\eta}(\gamma, D)=\sum_{\pi \in \mathcal{P}} F_{\pi} Q_{\eta, \pi}$ where $Q_{\eta, \pi} \in \mathbb{Z}\left[h_{0}, \ldots, \widehat{h}_{\eta}, \ldots, h_{r}\right]$ as in Lemma 4 .3. , and let $\Psi_{\omega}^{\prime} \in U\left(\mathfrak{h}_{\mathbb{C}}\right)$ be such that

$$
P_{\mathfrak{b}^{-}}\left(e_{i}^{(n)} Z_{\eta}\right)=\sum_{\omega \in \mathcal{P}} F_{\omega} \Psi_{\omega}^{\prime}
$$

Then $\Psi_{\omega}^{\prime} \in \mathbb{Z}\left[h_{0}, \ldots, h_{r}\right]$ and $\operatorname{deg}_{h_{\eta}} \Psi_{\omega}^{\prime} \leq D g_{\eta}$.

Proof. The first part is exactly the same as in the finite case [Fra88, Lemma 4.3]. Also,

$$
\begin{aligned}
\operatorname{deg}_{h_{\eta}} \Psi_{\omega}^{\prime} & =\max \left\{\operatorname{deg}_{h_{\eta}} \Psi_{\pi, \omega}+\operatorname{deg}_{h_{\eta}} Q_{\eta, \pi} \mid \operatorname{wt}(\pi)=D \gamma\right\} \\
& \leq \delta_{i, \eta} \max \left\{\pi\left(\alpha_{\eta}\right) \mid \operatorname{wt}(\pi)=D \gamma\right\} \leq D g_{\eta} .
\end{aligned}
$$

This proves (2).

Combining Lemma 4.2 and Lemma 4.5, we can express $P_{\mathfrak{b}^{-}}\left(e_{i}^{(n)} Z_{\eta}\right)$ as

$$
P_{\mathfrak{b}^{-}}\left(e_{i}^{(n)} Z_{\eta}\right)=\sum_{m=0}^{D g_{\eta}} u_{m}\left(\begin{array}{c}
h_{\gamma}+\rho\left(h_{\gamma}\right)-D \\
m
\end{array}\right)
$$

for some $u_{m} \in U\left(\mathfrak{n}_{\mathbb{Z}}^{-}\right) \otimes \mathbb{Z}\left[h_{0}, \ldots, \widehat{h}_{\eta}, \ldots, h_{r}\right] \otimes \mathbb{Z}_{(p)}$.

Proposition 4.6. Let $Z_{\eta}=Z_{\eta}(\gamma, D)$. If $\gamma=\sum g_{i} \alpha_{i} \in \Phi_{r e}^{+}$is $\eta$-good then $h_{\gamma}+$ $\rho\left(h_{\gamma}\right)-D$ divides $P_{\mathfrak{b}^{-}}\left(e_{i}^{(n)} Z_{\eta}\right)$, that is, $u_{0}$ defined in (4.1) equals to 0 .

Proof. For each $\chi \in H_{\gamma, D}$ we have $e_{i}^{(n)} Z_{\eta} v_{\chi}^{+}=0$ and hence $P_{\mathfrak{b}^{-}}\left(e_{i}^{(n)} Z_{\eta}\right) v_{\chi}^{+}=0$. Thus $h_{\gamma}+\rho(\gamma)-D$ divides $P_{\mathfrak{b}^{-}}\left(e_{i}^{(n)} Z_{\eta}\right)$. 
4.3. Homomorphisms between Verma modules. Now we are in a position to construct the homomorphisms in detail. Note that the naive map $M(\mu)_{\mathbb{K}} \rightarrow M(\lambda)_{\mathbb{K}}$ sending $v_{\mu}^{+} \otimes 1_{\mathbb{K}}$ to $Z_{\eta} v_{\lambda}^{+} \otimes 1_{\mathbb{K}}$ is not necessarily nonzero since $p$ may divide $Z_{\eta}(\lambda)$ in $U_{\mathbb{Z}}$. In our construction we send $v_{\mu}^{+} \otimes 1_{\mathbb{K}}$ to $\left(Z_{\eta} v_{\lambda}^{+}\right) / p^{f} \otimes 1_{\mathbb{K}}$ instead, where $p^{f}$ is the highest $p$-power dividing $Z_{\eta}(\lambda)$ in $U_{\mathbb{Z}}$.

Proposition 4.7. If $N \in \mathbb{Z}_{>0}$ divides $Z_{\eta}(\lambda)$ in $U_{\mathbb{Z}}$ then $N$ also divides each $u_{m}$ defined in (4.1).

Proof. It is exactly the same as in the finite case [Fra88, Proposition 5.1].

Corollary 4.8. Assume that $\mu \uparrow_{\gamma} \lambda$ (cf. \$2.7) and $\gamma=\sum g_{i} \alpha_{i} \in \Phi_{r e}^{+}$is $\eta$-good. If $D g_{\eta}<p^{e}$ then there is a nonzero homomorphism $M(\mu)_{\mathbb{K}} \rightarrow M(\lambda)_{\mathbb{K}}$.

Proof. Let $p^{f}$ be the highest $p$-power dividing $Z_{\eta}(\lambda)$ in $U_{\mathbb{Z}}$. Applying Lemma 4.6 and Proposition 4.7 with $N=p^{f}$, we have

$$
e_{i}^{(n)} \frac{Z_{\eta}(\lambda)}{p^{f}} v_{\lambda}^{+}=\sum_{m=1}^{D g_{\eta}} \frac{u_{m}}{p^{f}}\left(\begin{array}{c}
\left\langle\lambda+\rho, \gamma^{\vee}\right\rangle-D \\
m
\end{array}\right) v_{\lambda}^{+} .
$$

Since $D g_{\eta}<p^{e}$, each $m<p^{e}$ and hence each $\left(\begin{array}{c}\left\langle\lambda+\rho, \gamma^{\vee}\right\rangle-D \\ m\end{array}\right)=\left(\begin{array}{c}M p^{e} \\ m\end{array}\right) \in p \mathbb{Z}$. Therefore $e_{i}^{(n)} \frac{Z_{\eta}(\lambda)}{p^{f}} v_{\lambda}^{+} \otimes 1_{\mathbb{K}}=\overline{0}$ and the map $M(\mu)_{\mathbb{K}} \rightarrow M(\lambda)_{\mathbb{K}}$ sending $v_{\mu}^{+} \otimes 1_{\mathbb{K}}$ to $\frac{Z_{\eta}(\lambda)}{p^{f}} v_{\lambda}^{+} \otimes 1_{\mathbb{K}}$ is a homomorphism.

\section{Homomorphisms Between Weyl modules}

Now we start to deal with the Weyl modules using reduction modulo $p$. To construct an analogous homomorphism as in Section 4, we have to make sure that $Z_{\eta} \bar{v}_{\lambda}^{+}$is nonzero in $L(\lambda)_{\mathbb{Z}}$, which is not obvious. This is done by using the contravariant form and the Shapovalov factor formula (= Lemma 5.2). Finally we prove the existence of nonzero homomorphisms between Weyl modules whose highest weights are $\gamma$-linked if the two highest weights are close enough. In this section we mainly follow the outline given in [Fra81, while some adaptations are needed for the affine type.

5.1. Contravariant Forms. In this section we review some basic properties of the contravariant form mentioned in [Hum08, Sect. 3.14, 3.15] and [Fra81, §8]. A symmetric bilinear form $C: U \times U \rightarrow U\left(\mathfrak{h}_{\mathbb{C}}\right)$ is called contravariant if

$$
C\left(u \cdot v, v^{\prime}\right)=C\left(v, \tau(u) \cdot v^{\prime}\right) \text { for all } u \in U, v, v^{\prime} \in U .
$$

Proposition 5.1.

(a) Let $P_{\mathfrak{h}}: U \rightarrow U\left(\mathfrak{h}_{\mathbb{C}}\right)$ be the projection. Then $C(u, v)=P_{\mathfrak{h}}(\tau(u) v)$ is a nonzero contravariant form.

(b) The weight spaces are orthogonal to each other with respect to $C$, i.e., $C\left(U_{\lambda}, U_{\mu}\right)=0$ if $\lambda \neq \mu$.

(c) Let $C^{\lambda}: U \times U \rightarrow \mathbb{C}$ be the evaluation of $C$ at $\lambda$. Then

$$
R a d C^{\lambda}=\left\{u \in U \mid u \bar{v}_{\lambda}^{+}=0\right\} .
$$


(d) $C$ is non-degenerate on $U\left(\mathfrak{b}_{\mathbb{C}}^{-}\right)$.

(e) $\operatorname{deg} C\left(F_{\pi}, F_{\omega}\right) \leq \min \left(\operatorname{deg} F_{\pi}, \operatorname{deg} F_{\omega}\right)$.

5.2. Shapovalov Factor Formula. Recall that for arbitrary Kac-Moody algebras, the Shapovalov determinant formula [KK79, Theorem 1] computes the determinant of the contravariant form on $U_{-D \gamma}$ as follows.

$$
\left.\operatorname{det} C\right|_{U_{-D \gamma}}=\prod_{\alpha \in \Phi_{r e}^{+}} \prod_{n=1}^{\infty}\left(h_{\alpha}+\rho\left(h_{\alpha}\right)-n\right)^{P(D \gamma-n \alpha)} \prod_{\alpha \in \Phi_{i m}^{+}}\left(h_{\alpha}+\rho\left(h_{\alpha}\right)\right)^{\sum_{n=1}^{\infty} P(D \gamma-n \alpha)} .
$$

Here $P$ is the Kostant partition function. In this section we prove a variant that describes the common factor for the column corresponding to an integral Shapovalov element $Z(\gamma, D)$.

Lemma 5.2. Let $Z=Z(\gamma, D)$ for some $\gamma \in \Phi_{r e}^{+}$and $D \in \mathbb{Z}_{>0}$. Define $\epsilon_{i}, \gamma_{i}$ as in Lemma 3.7. Let $b_{i} \in \mathbb{Z}_{\geq 0}$ be such that $\gamma=\sum b_{i} \epsilon_{i}$, and let $\beta_{i}=s_{\epsilon_{1}} \cdots s_{\epsilon_{i-1}}\left(\epsilon_{i}\right) \in \Phi_{r e}^{+}$. Then for any $u \in U$ we have

$$
C(u, Z) \in \mathbb{C} \prod_{i=1}^{n} \prod_{j=1}^{D b_{i}}\left(h_{\beta_{i}}+\rho\left(h_{\beta_{i}}\right)-j\right) .
$$

Proof. We will show that for fixed $i \in\{1, \ldots, n\}$ and $j \in\left\{1, \ldots, D b_{i}\right\}$ that $h_{\beta_{i}}+$ $\rho\left(h_{\beta_{i}}\right)-j$ divides $C(u, Z)$. By Proposition 5.1 one needs to check that $C^{\lambda}(u, Z)=$ 0 for all $\lambda \in H_{\beta_{i}, j}$, equivalently, $Z v_{\lambda}^{+} \in N(\lambda)_{\mathbb{C}}$. So it remains to check that there is a dense subset $\Theta$ of $H_{\beta_{i}, j}$ such that for any $\lambda \in \Theta$, we have

$$
Z v_{\lambda}^{+} \in M\left(s_{\alpha} \cdot \lambda\right)_{\mathbb{C}}, \quad \alpha \in \Phi_{\mathrm{re}^{+}} .
$$

Now $q_{i}=-\left\langle\lambda+\rho, \beta_{i}^{\vee}\right\rangle=-j<0$. By assumption $q_{i}+D b_{i}=D b_{i}-j \geq 0$, and hence by Lemma 3.10 there is a commutative diagram of Verma module inclusions

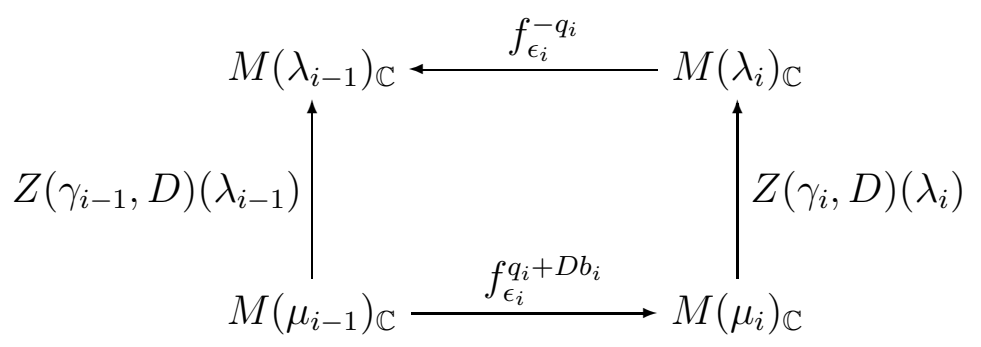

Recall that as in Remark 3.8 we can choose a dense subset of $H_{\gamma, M P^{e}+D}$ such that for any $\lambda$ in the subset we have the following commutative diagram of weights.

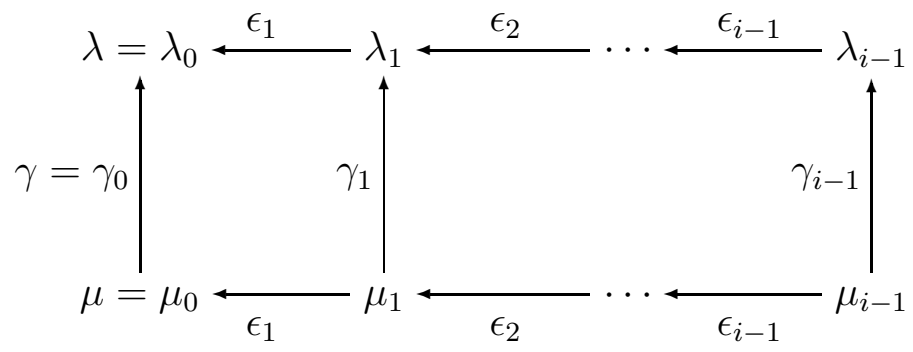


By Lemma 3.9 the above diagram leads to a commutative diagram of Verma module inclusions.

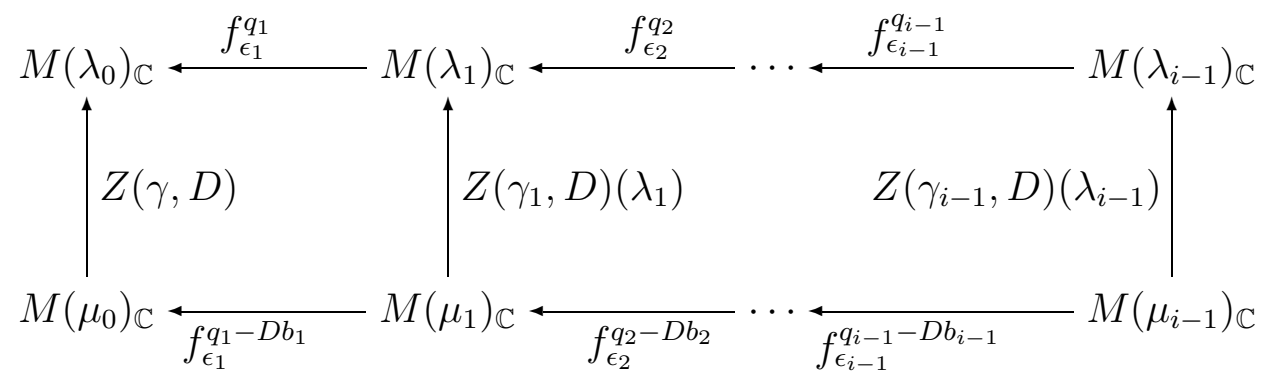

Finally, calculations show that if $i \neq n$ then for a suitable choice of dense subset of $H_{\gamma, M P^{e}+D}$ there is a commutative diagram of weights as the following with $\lambda_{i-1}^{\prime}=\lambda_{i}, \lambda_{k}^{\prime}=s_{\epsilon_{k+1}} \cdot \lambda_{k+1}^{\prime}, \mu_{i-1}^{\prime}=\mu_{i}, \mu_{k}^{\prime}=s_{\epsilon_{k+1}} \cdot \mu_{k+1}^{\prime}, \beta_{k}^{\prime}=s_{\epsilon_{k+1}} \cdots s_{\epsilon_{i-1}}\left(\epsilon_{i}\right)$, $\gamma_{i-1}^{\prime}=\gamma_{i}$ and $\gamma_{k}^{\prime}=s_{\epsilon_{k+1}}\left(\gamma_{k+1}\right)$.

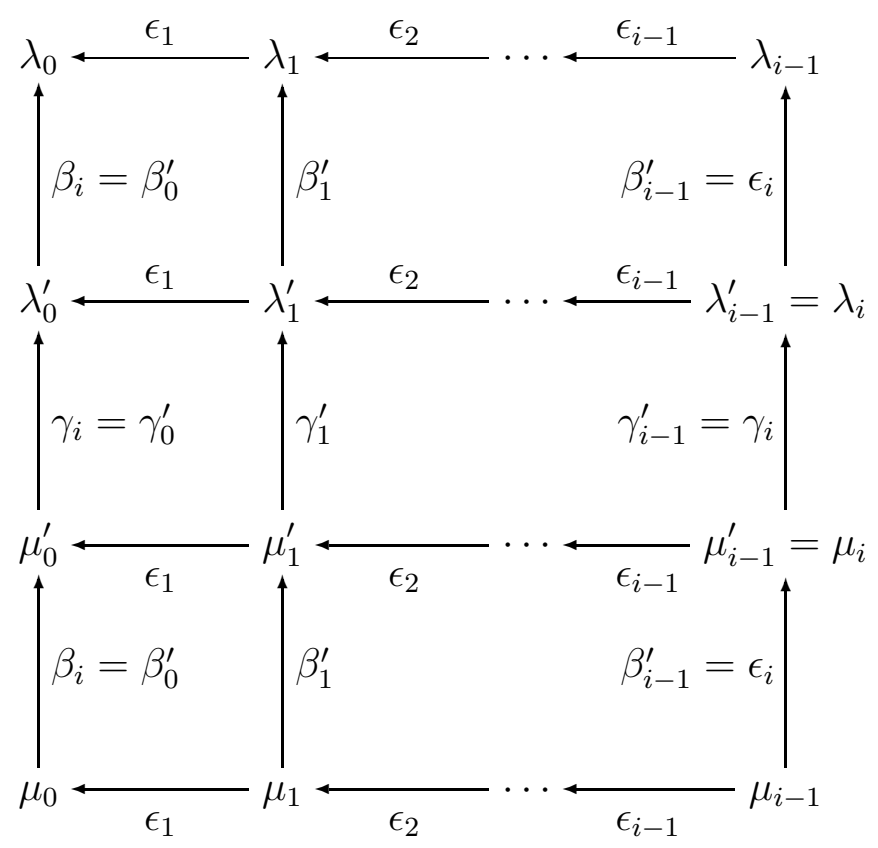


Now let $Q_{k}=\left\langle\gamma_{k}^{\prime}, \epsilon_{k}^{\vee}\right\rangle$. Again by Lemma 3.9 the above diagram leads to a commutative diagram of Verma module inclusions.

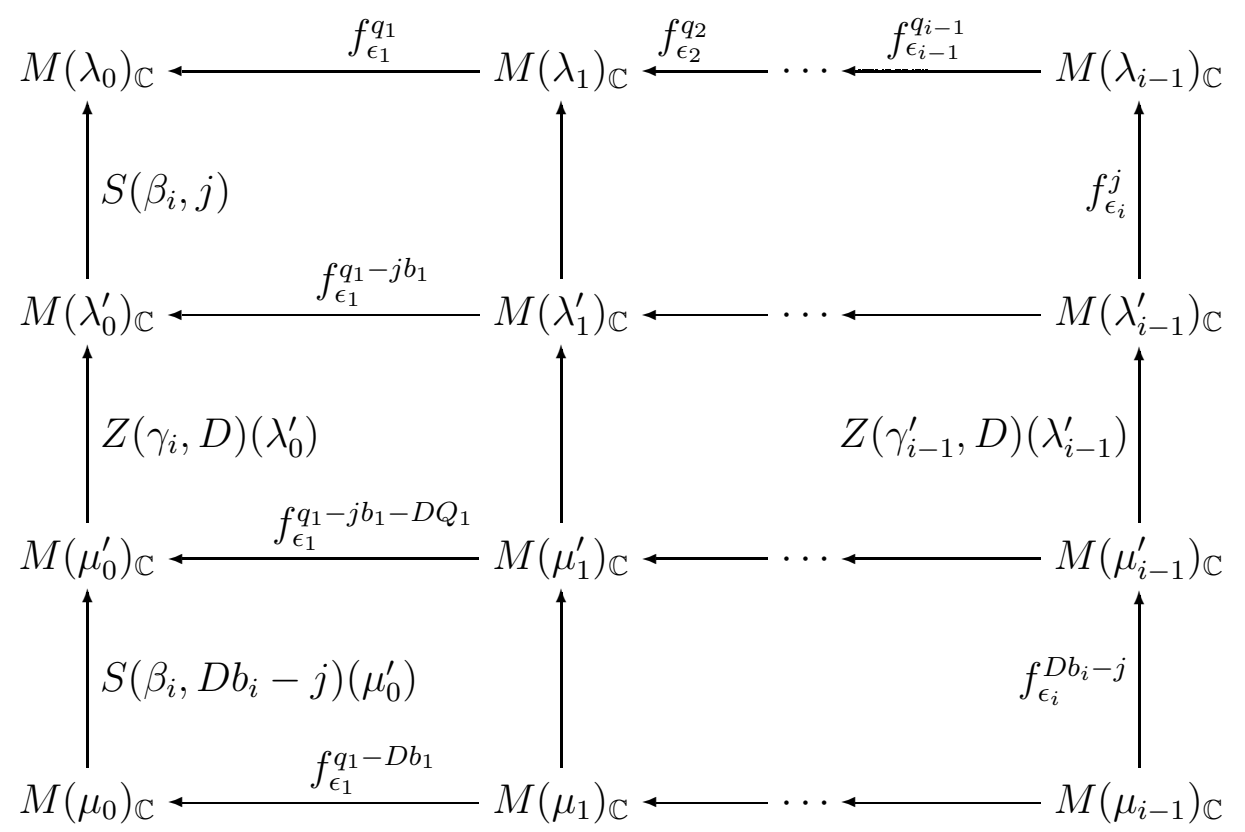

Combining Diagrams (5.1), (5.2) and (5.3) and using the fact that $U$ has no zero divisors, we obtain another commutative diagram below:

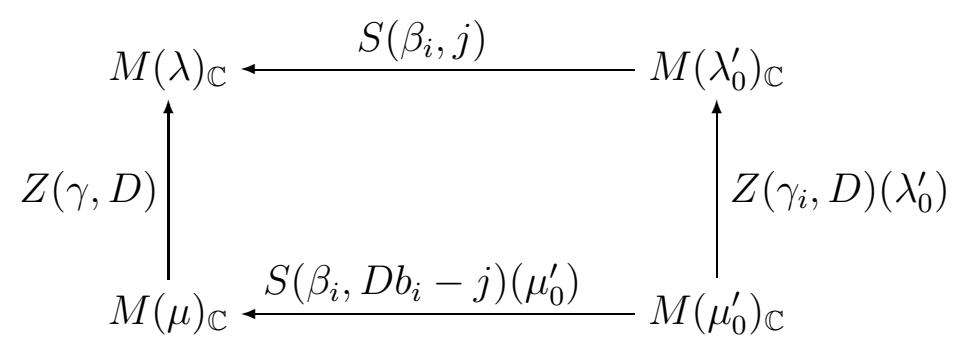

This shows that $Z v_{\lambda}^{+}=S\left(\beta_{i}, D b_{i}-j\right)\left(\mu_{0}^{\prime}\right) Z\left(\gamma_{i}, D\right)\left(\lambda_{0}^{\prime}\right) S\left(\beta_{i}, j\right) v_{\lambda}^{+} \in M\left(s_{\beta_{i}} \cdot \lambda\right)_{\mathbb{C}}$.

For the special case $i=n$, Diagram (5.3) is replaced by

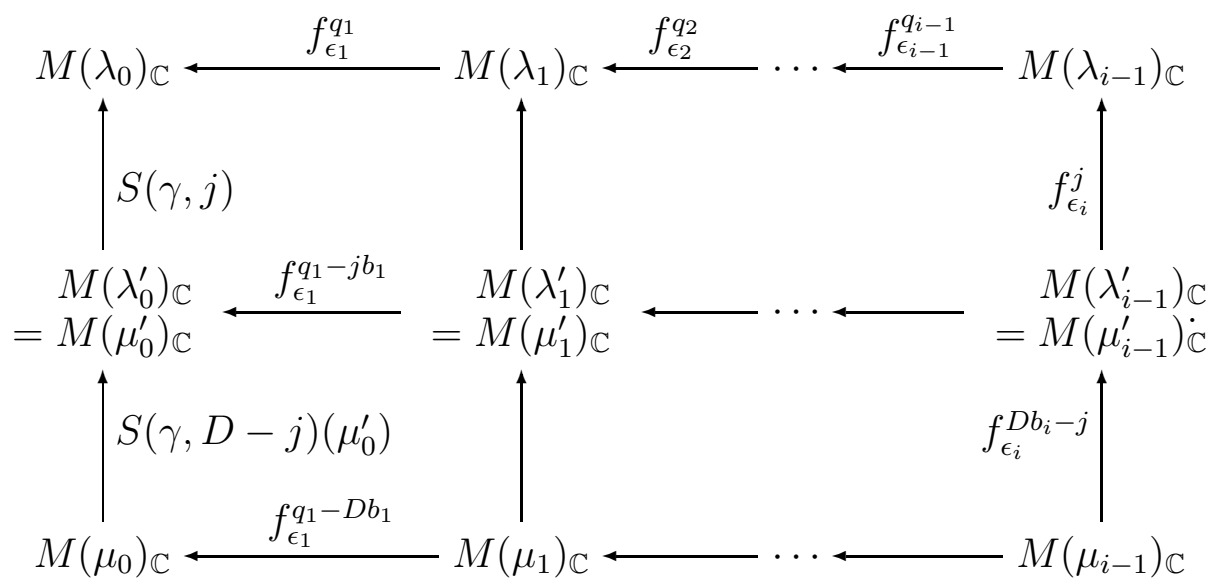


Similarly we have $Z v_{\lambda}^{+} \in M\left(s_{\beta_{n}} \cdot \lambda\right)_{\mathbb{C}}=M\left(s_{\gamma} \cdot \lambda\right)_{\mathbb{C}}$.

Note that the $\beta_{i}$ 's are distinct and none of them are multiples of others, so the factors $h_{\beta_{i}}+\rho\left(h_{\beta_{i}}\right)-j$ are relatively prime. Using Proposition 5.1(e), one can show that those are all the factors, and hence each $C(u, Z)$ is a scalar multiple of the product of these factors.

Corollary 5.3. If $\gamma$ is $\eta$-good then $Z_{\eta} \bar{v}_{\lambda}^{+} \neq \overline{0}$ in $L(\lambda)_{\mathbb{Z}}$.

Proof. Suppose that $Z_{\eta} \bar{v}_{\lambda}^{+}=\overline{0}$ in $L(\lambda)_{\mathbb{Z}}$, then $Z \bar{v}_{\lambda}^{+}=\overline{0}$ in $L(\lambda)_{\mathbb{Z}}$ as well. Therefore $C^{\lambda}(u, Z(\lambda))=0$ for all $u \in U$ and hence $C^{\lambda}(u, Z)=0$ for all $u \in U$. By Proposition 5.1 we know that $C$ is non-degenerate on $U\left(\mathfrak{b}_{\mathbb{C}}^{-}\right)$and hence the fact $Z \neq 0$ implies $C(u, Z) \neq 0$ for some $u \in U$.

Applying Lemma 5.2, we have $\prod_{i=1}^{n} \prod_{j=1}^{D b_{i}}\left(h_{\beta_{i}}+\rho\left(h_{\beta_{i}}\right)-j\right)(\lambda)=0$, and hence $\left\langle\lambda+\rho, \beta_{i}^{\vee}\right\rangle=j$ for some $1 \leq i \leq n$ and $1 \leq j \leq D b_{i}$. Therefore both $j$ and $D b_{i}-j$ are non-negative so that $\lambda_{i}$ and $\mu_{i}$ are on the opposite sides of the hyperplane $H_{\epsilon_{i}, 0}$. This shows that they are in different chambers, which is a contradiction.

5.3. BGG resolution. Kumar has generalized the strong BGG resolution over any Kac-Moody algebras.

Proposition 5.4. For each $\lambda \in X^{+}$there is an exact sequence of $\mathfrak{g}_{\mathbb{C}}$-modules:

$$
\cdots \rightarrow \mathcal{C}_{L} \rightarrow \cdots \rightarrow \mathcal{C}_{1} \rightarrow M(\lambda)_{\mathbb{C}} \rightarrow L(\lambda)_{\mathbb{C}} \rightarrow 0,
$$

where $\mathcal{C}_{L}=\bigoplus_{\substack{w \in W \\ l(w)=L}} M(w \cdot \lambda)_{\mathbb{C}}$

Proof. See [Kum90, Theorem 3.20].

Taking advantage of the BGG resolution, we describe bases of certain weight spaces of Verma modules and Weyl modules in characteristic 0.

Lemma 5.5. Assume that $\lambda \in X^{+}, \beta=\sum m_{i} \alpha_{i} \in Q^{+}$and $w \in W$. If there is an $\eta \in I$ such that $s_{\alpha_{\eta}}$ occurs in $w$ and $\left\langle\lambda+\rho, \alpha_{\eta}^{\vee}\right\rangle>m_{\eta}$, then $\lambda-\beta$ is not a weight of $M(w \cdot \lambda)_{\mathbb{C}}$. In particular, each $\mathcal{C}_{L}(c f$. Proposition 5.4) has weight space

$$
\left(\mathcal{C}_{L}\right)_{\lambda-\beta}=\bigoplus_{\substack{w \in W_{I \backslash\{\eta\}} \\ l(w)=L}} M(w \cdot \lambda)_{\mathbb{C}, \lambda-\beta},
$$

where $W_{I \backslash\{\eta\}}$ is the parabolic subgroup of $W$ generated by $\left\{s_{\alpha_{i}}\right\}_{i \in I \backslash\{\eta\}}$.

Proof. Fix a reduced expression $w=s_{\alpha_{i_{N}}} \cdots s_{\alpha_{i_{1}}}$ and let $\beta_{j}^{\prime}=s_{\alpha_{i_{N}}} \cdots s_{\alpha_{i_{j+1}}}\left(\alpha_{i_{j}}\right)$. Suppose that $\lambda-\beta$ is a weight of $M(w \cdot \lambda)_{\mathbb{C}}$, then

$$
\lambda-\beta<w \cdot \lambda=\lambda-\sum_{j=1}^{N}\left\langle\lambda+\rho, \alpha_{i_{j}}^{\vee}\right\rangle \beta_{j}^{\prime} .
$$


Since $s_{\alpha_{\eta}}$ occurs in $w$, there is an integer $L$ which is the largest one satisfying $\alpha_{\eta}=\alpha_{i_{L}}$, that is,

$$
\beta_{L}^{\prime}=s_{\alpha_{i_{N}}} \cdots s_{\alpha_{i_{L+1}}}\left(\alpha_{\eta}\right)=\alpha_{\eta}+\sum_{k \neq \eta} n_{k} \alpha_{k} \text { for some } n_{k} \geq 0 .
$$

Hence,

$$
\lambda-\beta<\lambda-\left\langle\lambda+\rho, \alpha_{i_{L}}^{\vee}\right\rangle \beta_{L}^{\prime}<\lambda-\left\langle\lambda+\rho, \alpha_{\eta}^{\vee}\right\rangle \alpha_{\eta},
$$

and therefore $\left\langle\lambda+\rho, \alpha_{\eta}^{\vee}\right\rangle<m_{\eta}$, which is a contradiction.

Assume that $\lambda \in X^{+}, w \in W$ with a fixed reduced expression $w=s_{\alpha_{i_{N}}} \cdots s_{\alpha_{i_{1}}}$. Let $\lambda_{0}=\lambda$, and let

$$
\lambda_{j}=s_{\alpha_{i_{j}}} \cdot \lambda_{j-1}, \quad \beta_{j}=s_{\alpha_{i_{1}}} \cdots s_{\alpha_{i_{j-1}}}\left(\alpha_{i_{j}}\right) \text { for } j=1, \ldots, N .
$$

Note that for each $j$, the map $M\left(\lambda_{j}\right)_{\mathbb{C}} \rightarrow M\left(\lambda_{j-1}\right)_{\mathbb{C}}$ sending $v_{\lambda_{j}}^{+}$to $S\left(\alpha_{i_{j}},\left\langle\lambda_{j-1}+\right.\right.$ $\left.\left.\rho, \alpha_{i_{j}}^{\vee}\right\rangle\right) v_{\lambda_{j-1}}^{+}$is an inclusion, where

$$
\left\langle\lambda_{j-1}+\rho, \alpha_{i_{j}}^{\vee}\right\rangle=\left\langle s_{\alpha_{i_{j-1}}} \cdots s_{\alpha_{i_{1}}}(\lambda+\rho), \alpha_{i_{j}}^{\vee}\right\rangle=\left\langle\lambda+\rho, \beta_{j}^{\vee}\right\rangle .
$$

Therefore, the composition

$$
M(w \cdot \lambda)_{\mathbb{C}} \rightarrow M\left(\lambda_{N-1}\right)_{\mathbb{C}} \rightarrow \cdots \rightarrow M\left(\lambda_{1}\right)_{\mathbb{C}} \rightarrow M(\lambda)_{\mathbb{C}}
$$

is again an inclusion sending $v_{w \cdot \lambda}^{+}$to $S v_{\lambda}^{+}$, where $S$ is a product of generic Shapovalov elements, given by

$$
S=S\left(\alpha_{i_{1}},\left\langle\lambda+\rho, \beta_{1}^{\vee}\right\rangle\right) \cdots S\left(\alpha_{i_{N}},\left\langle\lambda+\rho, \beta_{N}^{\vee}\right\rangle\right)=f_{\alpha_{i_{1}}}^{\left\langle\lambda+\rho, \beta_{1}^{\vee}\right\rangle} \cdots f_{\alpha_{i_{N}}}^{\left\langle\lambda+\rho, \beta_{N}^{\vee}\right\rangle} .
$$

Lemma 5.6. Assume that $\lambda \in X^{+}, w \in W_{I \backslash\{\eta\}}$ and $\beta \in Q^{+}$. Then for each $k \in \mathbb{Z}_{\geq 0}$,

$$
\left\{F_{\pi} S v_{\lambda+k \varpi_{\eta}}^{+} \mid \operatorname{wt}(\pi)=w \cdot \lambda-(\lambda-\beta)\right\}
$$

is a basis of the image of $M\left(w \cdot \lambda+k \varpi_{\eta}\right)_{\mathbb{C}}$ in $M\left(\lambda+k \varpi_{\eta}\right)_{\mathbb{C}}$, where $S$ is as defined in (5.6).

Proof. Fix a reduced expression $w=s_{\alpha_{i_{N}}} \cdots s_{\alpha_{i_{1}}}$ and define $\lambda_{j}, \beta_{j}$ as in (5.5). Since $w \in W_{I \backslash\{\eta\}}$, we have $w\left(\varpi_{\eta}\right)=\varpi_{\eta}$ and hence for each $k \in \mathbb{Z}$ and $j=1, \ldots, N$,

$$
w \cdot\left(\lambda_{j}+k \varpi_{\eta}\right)=w \cdot \lambda_{j}+w\left(k \varpi_{\eta}\right)=w \cdot \lambda_{j}+k \varpi_{\eta} .
$$

By definition of generic Shapovalov elements, the map $M\left(\lambda_{j}+k \varpi_{\eta}\right)_{\mathbb{C}} \rightarrow M\left(\lambda_{j-1}+\right.$ $\left.k \varpi_{\eta}\right)_{\mathbb{C}}$ sending $v_{\lambda_{j}+k \varpi_{\eta}}^{+}$to $S\left(\alpha_{i_{j}},\left\langle\lambda+\rho, \beta_{j}^{\vee}\right\rangle\right) v_{\lambda_{j-1}+k \varpi_{\eta}}^{+}$is an inclusion for each $j$, which leads to another inclusion $M\left(w \cdot \lambda+k \varpi_{\eta}\right)_{\mathbb{C}} \rightarrow M\left(\lambda+k \varpi_{\eta}\right)_{\mathbb{C}}$ sending $v_{w \cdot \lambda+k \varpi_{\eta}}^{+}$ to $S v_{\lambda}^{+}+k \varpi_{\eta}$, where $S$ is exactly the same element defined in (5.6).

Lemma 5.7. Assume that $\left\{B_{i} \bar{v}_{\lambda}^{+}\right\}_{i \in \Lambda}$ is a basis of $L(\lambda)_{\mathbb{C}, \lambda-\beta}$ with $B_{i} \in U\left(\mathfrak{n}_{\mathbb{C}}^{-}\right)$for some $\lambda \in X^{+}$and $\beta=\sum m_{i} \alpha_{i} \in Q^{+}$. If $\left\langle\lambda+\rho, \alpha_{\eta}^{\vee}\right\rangle>m_{\eta}$, then for each $k \in \mathbb{Z}_{\geq 0}$,

$$
\left\{B_{i} \bar{v}_{\lambda+k \varpi_{\eta}}^{+}\right\}_{i \in \Lambda}
$$


is a basis of $L\left(\lambda+k \varpi_{\eta}\right)_{\mathbb{C}, \lambda+k \varpi_{\eta}-\beta}$ satisfying the property (5.7) below: for each $y \in U\left(\mathfrak{n}_{\mathbb{C}}^{-}\right)_{-\beta}$ and $k \in \mathbb{Z}_{\geq 0}$, there are $C_{i}^{(y)} \in \mathbb{C}$ (independent of $k$ ) such that

$$
y \bar{v}_{\lambda+k \varpi_{\eta}}^{+}=\sum_{i \in \Lambda} C_{i}^{(y)} B_{i} \bar{v}_{\lambda+k \varpi_{\eta}}^{+} .
$$

Proof. By Lemma 5.5 and Lemma 5.6, there is a basis $\left\{B_{j}^{\prime} \bar{v}_{\lambda}^{+}\right\}_{j \in \Lambda^{\prime}}$ of $L(\lambda)_{\mathbb{C}, \lambda-\beta}$ with $B_{j}^{\prime} \in U\left(\mathfrak{n}_{\mathbb{C}}^{-}\right)$satisfying Property (5.7), since $F_{\pi} S$ is independent of $k$. Plugging in $y=\sum_{i \in \Lambda} C_{i} B_{i}$ for arbitrary $C_{i} \in \mathbb{C}$ to (5.7), we obtain that

$$
\sum_{i \in \Lambda} C_{i} B_{i} \bar{v}_{\lambda+k \varpi_{\eta}}^{+}=\sum_{j \in \Lambda^{\prime}} C_{j}^{\prime} B_{j}^{\prime} \bar{v}_{\lambda+k \varpi_{\eta}}^{+}
$$

where $C_{j}^{\prime}=C_{j}^{\left(\sum_{i \in \Lambda} C_{i} B_{i}\right)}$.

Note that $\sum_{j \in \Lambda^{\prime}} C_{j}^{\prime} B_{j}^{\prime} \bar{v}_{\lambda+k \varpi_{\eta}}^{+}=0$ if and only if all the $C_{j}^{\prime}$ 's are zero, which is equivalent to that all $C_{i}$ 's are zero. Therefore $\left\{B_{i} \bar{v}_{\lambda+k \varpi_{\eta}}^{+}\right\}_{i \in \Lambda}$ is a basis of $L(\lambda+$ $\left.k \varpi_{\eta}\right)_{\mathbb{C}, \lambda+k \varpi_{\eta}-\beta}$ for each $k \in \mathbb{Z}_{\geq 0}$. Moreover, $\left\{B_{i} \bar{v}_{\lambda}^{+}\right\}_{i \in \Lambda}$ also has Property (5.7).

5.4. Homomorphisms between Weyl Modules. Here we fix $\lambda, \mu=\lambda-D \gamma \in$ $X^{+}$satisfying $\mu \uparrow_{\gamma} \lambda$ for some $\eta$-good positive real root $\gamma=\sum g_{i} \alpha_{i}$ and $D \in$ $\mathbb{Z}_{>0}$. Fix $Z_{\eta}=Z_{\eta}(\gamma, D)$ to be an $h_{\eta}$-avoiding integral Shapovalov element. Fix a basis $\left\{B_{a} \bar{v}_{\lambda}^{+}\right\}_{a \in \mathcal{B}_{1}}$ of $L(\lambda)_{\mathbb{Z}, \mu}$ with $B_{a} \in U\left(\mathfrak{n}_{\mathbb{Z}}^{-}\right)_{-D \gamma}$, and fix a basis $\left\{B_{b}^{\prime} \bar{v}_{\lambda}^{+}\right\}_{b \in \mathcal{B}_{2}}$ of $L(\lambda)_{\mathbb{Z}, \mu+n \alpha_{i}}$ for some fixed $i \in I$ and $n \in \mathbb{Z}_{\geq 0}$, with $B_{b}^{\prime} \in U\left(\mathfrak{n}_{\mathbb{Z}}^{-}\right)_{-D \gamma+n \alpha_{i}}$.

We start with a corollary of Lemma 5.7 .

Corollary 5.8. If $\left\langle\lambda+\rho, \alpha_{\eta}^{\vee}\right\rangle>D g_{\eta}$, then for each $k \in \mathbb{Z}_{\geq 0}$ we have

$$
e_{i}^{(n)} Z_{\eta} \bar{v}_{\lambda+k \varpi_{\eta}}^{+}=\sum_{m=1}^{D g_{\eta}} \sum_{b \in \mathcal{B}_{2}} u_{b, m}(\lambda)\left(\begin{array}{c}
\left\langle\lambda+k \varpi_{\eta}+\rho, \gamma^{\vee}\right\rangle-D \\
m
\end{array}\right) B_{b}^{\prime} \bar{v}_{\lambda+k \varpi_{\eta}}^{+}
$$

for some polynomials $u_{b, m} \in \mathbb{C}\left[h_{0}, \ldots, \widehat{h}_{\eta}, \ldots, h_{r}\right]$.

Proof. Applying Lemma 5.7with $\beta=D \gamma-n \alpha_{i}$ and $y=F_{\pi}$ with wt $(\pi)=D \gamma-n \alpha_{i}$, we have, for each $k \in \mathbb{Z}_{\geq 0}$,

$$
F_{\pi} \bar{v}_{\lambda+k \varpi_{\eta}}^{+}=\sum_{b \in \mathcal{B}_{2}} C_{b}^{\left(F_{\pi}\right)} B_{b}^{\prime} \bar{v}_{\lambda+k \varpi_{\eta}}^{+}
$$

where $C_{b}^{\left(F_{\pi}\right)}$ is independent of $k$. Let $u_{m} \in U\left(\mathfrak{n}_{\mathbb{Z}}^{-}\right) \otimes \mathbb{C}\left[h_{0}, \ldots, \widehat{h}_{\eta}, \ldots, h_{r}\right]$ be as defined in (4.1). We have

$$
u_{m}=\sum_{\pi \in \mathcal{P}} F_{\pi} u_{m, \pi},
$$


for some $u_{m, \pi} \in \mathbb{C}\left[h_{0}, \ldots, \widehat{h}_{\eta}, \ldots, h_{r}\right]$, that is, $u_{m, \pi}\left(\lambda+k \varpi_{\eta}\right)=u_{m, \pi}(\lambda)$ for any $k \in \mathbb{Z}$. Thus, by Proposition 4.6, we have

$$
\begin{aligned}
& e_{i}^{(n)} Z_{\eta} \bar{v}_{\lambda+k \varpi_{\eta}}^{+}=\sum_{m=1}^{D g_{\eta}} u_{m}\left(\begin{array}{c}
h_{\gamma}+\rho\left(h_{\gamma}\right)-D \\
m
\end{array}\right) \bar{v}_{\lambda+k \varpi_{\eta}}^{+} \\
& =\sum_{m=1}^{D g_{\eta}} \sum_{\pi \in \mathcal{P}} u_{m, \pi}(\lambda)\left(\begin{array}{c}
\left\langle\lambda+k \varpi_{\eta}+\rho, \gamma^{\vee}\right\rangle-D \\
m
\end{array}\right) F_{\pi} \bar{v}_{\lambda+k \varpi_{\eta}}^{+} \\
& =\sum_{m=1}^{D g_{\eta}} \sum_{b \in \mathcal{B}_{2}}\left(\sum_{\pi \in \mathcal{P}} u_{m, \pi}(\lambda) C_{b}^{\left(F_{\pi}\right)}\right)\left(\underset{m}{\left\langle\lambda+k \varpi_{\eta}+\rho, \gamma^{\vee}\right\rangle-D}\right) B_{b}^{\prime} \bar{v}_{\lambda+k \varpi_{\eta}}^{+} .
\end{aligned}
$$

Let $u_{m, b}=\sum_{\pi \in \mathcal{P}} u_{m, \pi} C_{b}^{\left(F_{\pi}\right)}$ and we are done.

Lemma 5.9. If $\left\langle\lambda+\rho, \alpha_{\eta}^{\vee}\right\rangle>D g_{\eta}$, then for each $k \in \mathbb{Z}_{\geq 0}$, we have

$$
e_{i}^{(n)} Z_{\eta} \bar{v}_{\lambda+k \varpi_{\eta}}^{+}=\sum_{m=1}^{D g_{\eta}} \sum_{\substack{a \in \mathcal{B}_{1} \\ b \in \mathcal{B}_{2}}} C_{a} C_{a, b, m}(\lambda)\left(\underset{m}{\left\langle\lambda+k \varpi_{\eta}+\rho, \gamma^{\vee}\right\rangle-D}\right) B_{b}^{\prime} \bar{v}_{\lambda+k \varpi_{\eta}}^{+},
$$

for some $C_{a} \in \mathbb{C}$ and polynomials $C_{a, b, m} \in \mathbb{C}\left[h_{0}, \ldots, \widehat{h}_{\eta}, \ldots, h_{r}\right]$, which are independent of $k$.

Proof. Now let $Z_{\eta} \bar{v}_{\lambda}^{+}=\sum_{a \in \mathcal{B}_{1}} C_{a} B_{a} \bar{v}_{\lambda}^{+}$with $C_{a} \in \mathbb{C}$. Applying Lemma 5.7 with $\beta=D \gamma-n \alpha_{i}$ and $y=e_{i}^{(n)} B_{a}$, we have, for each $k \in \mathbb{Z}_{\geq 0}$,

$$
e_{i}^{(n)} B_{a} \bar{v}_{\lambda+k \varpi_{\eta}}^{+}=\sum_{b \in \mathcal{B}_{2}} C_{b}^{\left(e_{i}^{(n)} B_{a}\right)} B_{b}^{\prime} \bar{v}_{\lambda+k \varpi_{\eta}}^{+}
$$

where each $C_{b}^{\left(e_{i}^{(n)} B_{a}\right)}$ is independent of $k$. Similar to the proof of Corollary 5.8, one obtains that

$$
e_{i}^{(n)} B_{a} \bar{v}_{\lambda+k \varpi_{\eta}}^{+}=\sum_{m \geq 0} \sum_{b \in \mathcal{B}_{2}} C_{a, b, m}(\lambda)\left(\underset{m}{\left\langle\lambda+k \varpi_{\eta}+\rho, \gamma^{\vee}\right\rangle-D}\right) B_{b}^{\prime} \bar{v}_{\lambda+k \varpi_{\eta}}^{+},
$$

for some $C_{a, b, m} \in \mathbb{C}\left[h_{0}, \ldots, \widehat{h}_{\eta}, \ldots, h_{r}, d\right]$ that is independent of $k$. Therefore

$$
e_{i}^{(n)} Z_{\eta} \bar{v}_{\lambda+k \varpi_{\eta}}^{+}=\sum_{m \geq 0} \sum_{\substack{a \in \mathcal{B}_{1} \\ b \in \mathcal{B}_{2}}} C_{a} C_{a, b, m}(\lambda)\left({ }_{m}^{\left\langle\lambda+k \varpi_{\eta}+\rho, \gamma^{\vee}\right\rangle-D}\right) B_{b}^{\prime} \bar{v}_{\lambda+k \varpi_{\eta}}^{+} .
$$

Equating the coefficient of each $B_{b}^{\prime} \bar{v}_{\lambda+k \varpi_{\eta}}^{+}$on (5.8) and (5.9), one gets

$$
\sum_{m \geq 0} \sum_{a \in \mathcal{B}_{1}} C_{a} C_{a, b, m}(\lambda)\left(\begin{array}{c}
\left\langle\lambda+k \varpi_{\eta}+\rho, \gamma^{\vee}\right\rangle-D \\
m
\end{array}\right)=\sum_{m=1}^{D g_{\eta}} u_{b, m}(\lambda)\left(\underset{m}{\left\langle\lambda+k \varpi_{\eta}+\rho, \gamma^{\vee}\right\rangle-D}\right) \in \mathbb{C}[k] .
$$


Since the right hand side has finite degree in $k$, we may equate the coefficients of powers of $k$, and hence

$$
\sum_{a \in \mathcal{B}_{1}} C_{a} C_{a, b, m}(\lambda)= \begin{cases}u_{b, m}(\lambda) & \text { if } 1 \leq m \leq D g_{\eta} \\ 0 & \text { if } m=0 \text { or } m>D g_{\eta}\end{cases}
$$

Now we can prove our main theorem.

Theorem 5.10. Assume that $\mu \uparrow_{\gamma}^{e} \lambda$ and $\gamma=\sum g_{i} \alpha_{i} \in \Phi_{r e}^{+}$is $\eta$-good. If $D g_{\eta}<$ $\left\langle\lambda+\rho, \alpha_{\eta}^{\vee}\right\rangle$ and $D g_{\eta}<p^{e}$ then there exists a nonzero homomorphism $V(\mu) \rightarrow V(\lambda)$ between Weyl modules.

Proof. Let $p^{g}$ be the highest $p$-power dividing $Z_{\eta} \bar{v}_{\lambda}^{+}=\sum_{a \in \mathcal{B}_{1}} C_{a} B_{a} \bar{v}_{\lambda}^{+}$in $L(\lambda)_{\mathbb{Z}}$, that is, $C_{a} \in p^{g} \mathbb{Z}$ for each $a$. We shall show that the map sending $\bar{v}_{\mu}^{+} \otimes 1_{\mathbb{K}}$ to $\frac{Z_{\eta} \bar{v}_{\lambda}^{+}}{p^{g}} \otimes 1_{\mathbb{K}}$ is a homomorphism. Applying Lemma [5.9, we have

$$
e_{i}^{(n)} \frac{Z_{\eta} \bar{v}_{\lambda}^{+}}{p^{g}}=\sum_{m=1}^{D g_{\eta}} \sum_{\substack{a \in \mathcal{B}_{1} \\ b \in \mathcal{B}_{2}}} \frac{C_{a}}{p^{g}} C_{a, b, m}(\lambda)\left(\underset{m}{\left\langle\lambda+\rho, \gamma^{\vee}\right\rangle-D}\right) B_{b}^{\prime} \bar{v}_{\lambda}^{+} .
$$

Since $D g_{\eta}<p^{e}$, each $m<p^{e}$ and hence each $\left(\begin{array}{c}\left\langle\lambda+\rho, \gamma^{\vee}\right\rangle-D \\ m\end{array}\right)=\left(\begin{array}{c}M p^{e} \\ m\end{array}\right) \in p \mathbb{Z}$. Therefore $e_{i}^{(n)} \frac{Z_{\eta} \bar{v}_{\lambda}^{+}}{p^{g}} \otimes 1_{\mathbb{K}}=\overline{0}$ in $V(\lambda)$.

Example 5.11. In Example 4.4 we have $\gamma=2 \alpha_{0}+\alpha_{1}=2 \delta-\alpha_{1}=2 \varpi_{0}-2 \varpi_{1}+2 \delta$ is 1 -good for any prime with $g_{1}=1$ and

$$
\begin{aligned}
& Z_{1}=f_{1} f_{0}^{2}-f_{\delta, 1} f_{0}\left(h_{0}-1\right)+f_{\gamma}\left(h_{0}^{2}-h_{0}\right) \\
& =f_{1} f_{0}^{(2)}\left(2 h_{0}^{2}\right)-f_{0} f_{1} f_{0}\left(2 h_{0}^{2}-h_{0}-1\right)+f_{0}^{(2)} f_{1}\left(2 h_{0}^{2}-2 h_{0}\right) .
\end{aligned}
$$

For $\lambda=2 \varpi_{0}+\varpi_{1}$, then $\mu=\lambda-D \gamma \in X^{+}$implies $D=1$ and $\mu=3 \varpi_{1}-2 \delta$. Note that $\left\langle\lambda+\rho, \gamma^{\vee}\right\rangle=8=M p^{e}+D$. Hence $p=7$ is the only possible prime with $M=e=1$. Also, the assumptions are satisfied since $D g_{1}=1\left\langle\left\langle\lambda+\rho, \alpha_{1}^{\vee}\right\rangle=2\right.$ and $D g_{1}=1<p^{e}=7$. Note that

$$
Z_{1} \bar{v}_{\lambda}^{+}=\left(8 f_{1} f_{0}^{(2)}-5 f_{0} f_{1} f_{0}+4 f_{0}^{(2)} f_{1}\right) \bar{v}_{\lambda}^{+},
$$

so $g=0$ and therefore the map $V\left(3 \varpi_{1}-2 \delta\right) \rightarrow V\left(2 \varpi_{0}+\varpi_{1}\right)$ sending $\bar{v}_{\mu}^{+} \otimes 1_{\mathbb{K}}$ to $Z_{1} \bar{v}_{\lambda}^{+} \otimes 1_{\mathbb{K}}$ is a nonzero homomorphism.

\section{A Conjectural Strong Linkage PRinciple}

6.1. Formulation of a conjecture. Having in mind the strong linkage principles for classical cases, we formulate a conjecture (joint with W. Wang) on the strong linkage principle for the modular representation of affine Lie algebras. 
Conjecture 6.1 (Strong linkage principle). Let $\mu, \lambda \in X^{+}$, and let $L(\mu)$ be the unique irreducible highest weight $U_{\mathbb{K}}$-module with highest weight $\mu$. If $L(\mu)$ is a composition factor of the Weyl module $V(\lambda)$, then $\mu=\lambda$ or $\mu=\mu_{0} \uparrow \cdots \uparrow \mu_{N}=\lambda$ for some $N$ and $\mu_{i} \in X$ where

$$
x \uparrow y \Leftrightarrow \text { there exists }\left\{\begin{array} { l } 
{ n \in \mathbb { Z } _ { > 0 } , } \\
{ m \in \mathbb { Z } , } \\
{ \beta \in \Phi ^ { + } }
\end{array} \quad \text { such that } \left\{\begin{array}{l}
y-x=(n-m p) \beta \in Q^{+}, \\
n(\beta, \beta)=2(y+\rho, \beta) \in \mathbb{Z} / p \mathbb{Z} .
\end{array}\right.\right.
$$

Remark 6.2. If we interpret $p=0$, condition (6.1) coincides with Condition (2.3). For finite types, Condition (6.1) describes exactly the strong linkage for modular finite case below:

$$
x \uparrow y \Leftrightarrow \text { there exists }\left\{\begin{array}{l}
m \in \mathbb{Z}, \\
\beta \in \Phi^{+}
\end{array} \quad \text { such that } x=s_{\beta, m p} \cdot y<y .\right.
$$

Remark 6.3. The formulation of Conjecture6.1 also makes sense for all symmetrizable Kac-Moody algebras.

6.2. Candidates for reducible Weyl modules. Now we focus on the reducibility problem for Weyl modules. Note that we do not know the irreducibility for any Weyl module, but our main theorem is able to detect if a Weyl module is reducible.

The candidates for the high weights of reducible Weyl modules are those dominant integral weights which are $\gamma$-mirrored by another dominant integral weight. Note that if $\lambda, \mu \in X^{+}$such that $\mu \uparrow_{\gamma} \lambda$ for some positive real root $\gamma$, there is a unique weight $\lambda+t \delta \in X^{+}$such that $\mu+t \delta \uparrow_{\gamma} \lambda+t \delta$ and $\xi=0$ if we express $\lambda+t \delta=\sum_{i} \xi_{i} \varpi_{i}+\xi \delta$. Without loss of generality, we only need to consider the set defined below:

$$
Y^{+}=\left\{\lambda \in \sum_{i} \mathbb{Z}_{\geq 0} \varpi_{i} \mid \exists \mu \in X^{+} \text {s.t. } \mu \uparrow_{\gamma} \lambda \text { for some } \gamma \in \Phi_{\mathrm{re}}^{+}\right\} .
$$

Lemma 6.4. For an arbitrary affine type, the set of positive real roots can be described as the following.

$$
\Phi_{r e}^{+}=\bigcup_{i=1}^{3}\left\{\gamma_{0}+t \delta \mid \gamma_{0} \in \Phi_{i}^{+}, t \in i \mathbb{Z}_{\geq 0}\right\}
$$

where $\Phi_{i}^{+}$are subsets of $\Phi^{+}$for $i=1,2,3$.

In particular, $\Phi_{3}^{+}=\varnothing$ unless for type $\widetilde{D}_{4}^{(3)} . \Phi_{2}^{+}=\varnothing$ unless for type $\widetilde{A}_{r}^{(2)}, \widetilde{D}_{r}^{(2)}$ and $\widetilde{E}_{6}^{(2)}$. For untwisted types we have $\Phi_{1}^{+}=\left\{\alpha, \delta-\alpha \mid \alpha \in \Phi_{0}^{+}\right\}$.

Proof. This is done by a case-by-case analysis on the data of root systems.

Proposition 6.5. Let $\lambda \in X^{+}$be a dominant integral weight of fixed level $\ell$, and let $\gamma_{0} \in \Phi_{i}^{+}$for some $i$ as defined in Lemma 6.4. Let $C_{i}=\frac{2 i}{\left(\gamma_{0}, \gamma_{0}\right)}\left(\ell+h^{\vee}\right)$. If $\lambda-\operatorname{gcd}\left(C_{i}, p\right) \gamma_{0} \in X^{+}$then $\lambda \in Y^{+}$. 
Proof. For any positive real root of the form $\gamma=\gamma_{0}+i t \delta$ we have $(\gamma, \gamma)=\left(\gamma_{0}, \gamma_{0}\right)$, and hence for any $\lambda \in X^{+}$we have $\left\langle\lambda+\rho, \gamma^{\vee}\right\rangle=\left\langle\lambda+\rho, \gamma_{0}^{\vee}\right\rangle+C_{i}$ t. Therefore for $m, e \in \mathbb{Z}_{>0}$ we have

$$
s_{\gamma, M p^{e}} \cdot \lambda=s_{\gamma_{0}, C_{i} t+M p^{e}} \cdot \lambda-\left(\left\langle\lambda+\rho, \gamma^{\vee}\right\rangle-M p^{e}\right) t \delta .
$$

Therefore, we can always choose $t, M \in \mathbb{Z}_{>0}$ large enough such that

$$
s_{\gamma_{0}+i t \delta, M p^{e}} \cdot \lambda=\lambda-\operatorname{gcd}\left(C_{i}, p^{e}\right) \gamma_{0}-\left(\left\langle\lambda+\rho, \gamma^{\vee}\right\rangle-M p^{e}\right) t \delta
$$

By assumption $s_{\gamma_{0}+t \delta, M p^{e}} \cdot \lambda \in X^{+}$and hence $\lambda \in Y^{+}$.

Using Proposition 6.5, one can describe for each type and for each prime $p$ the lowest level weights in $Y^{+}$. For most types, every weight in $Y^{+}$has level $\geq 2$. $Y^{+}$ contains a level one weight if and only if the following conditions hold:

(1) $p$ is a odd prime that does not divide $h^{\vee}+1$.

(2) $\mathfrak{g}_{\mathbb{C}}$ is of type $\widetilde{B}_{r}, \widetilde{C}_{r}, \widetilde{F}_{4}$ or $\widetilde{G}_{2}$.

In this case, the level one weights are given by Table 1 below.

TABLE 1. The complete list of weights of level one in $Y^{+}$.

\begin{tabular}{|c|c|c|c|c|}
\hline Type & $\widetilde{B}_{r} ; r \geq 3$ & $\widetilde{C}_{r} ; r \geq 2$ & $\widetilde{F}_{4}$ & $\widetilde{G}_{2}$ \\
\hline weight & $\varpi_{0}, \varpi_{1}$ & $\varpi_{0}, \varpi_{r}$ & $\varpi_{0}$ & $\varpi_{0}, \varpi_{2}$ \\
\hline
\end{tabular}

This shows that for (possibly twisted) affine ADE types, there is no dominant integral weights that are $\gamma$-mirrored to $\varpi_{0}$ for any $\gamma \in \Phi_{\mathrm{re}}^{+}$. One might expect that the lack of $\gamma$-mirrored weights would imply that the basic representation is irreducible. However, the table in [BK02, §1] shows that for each (possibly twisted) affine ADE type, there exists a reducible basic representation for some prime $p \leq h$.

6.3. Quasi-simple weights. Our main theorem shows that the corresponding Weyl module of a weight in $Y^{+}$is reducible if the restrictions in Theorem 5.10 are satisfied. Here we shall demonstrate that these restrictions are actually quite mild.

Definition 6.6. A weight $\lambda \in Y^{+}$is called quasi-simple if Theorem 5.10 does not apply for any $\mu \in X^{+}$that is $\gamma$-mirrored to $\lambda$.

Note that every weight of level one described in Remark 6.5 is quasi-simple. Unfortunately, it seems that there is not an obvious pattern for the quasi-simple weights in general. 
6.4. Type $\widetilde{A}_{1}$. Assume that $\gamma=\alpha_{0}+t \delta$ with $t \geq 0$. Let $\left(\xi_{0}, \xi_{1}\right)$ be the shorthand notation of the weight $\xi_{0} \varpi_{0}+\xi_{1} \varpi_{1}$. Our main theorem has the following corollary.

Corollary 6.7. For type $\widetilde{A}_{1}$ and a fixed prime $p$, a weight $\lambda=\left(\xi_{0}, \ell-\xi_{0}\right)$ of level $\ell$ lies in $Y^{+}$if and only if there are integers $M, D, e \in \mathbb{Z}_{>0}$ and $t \in \mathbb{Z}_{\geq 0}$ satisfying the following conditions.

(1) $(\ell+2) t+\xi_{0}+1=M p^{e}+D$.

(2) $2 D \leq \xi_{0} \leq \ell$.

In particular, if $\lambda \in Y^{+}$, the corresponding Weyl module $V(\lambda)$ is reducible if either Condition (i) or (ii) below holds:

(i) $\operatorname{gcd}(t+1, p)=1$ and $D(t+1)<\min \left(\xi_{0}+1, p^{e}\right)$.

(ii) $\operatorname{gcd}(t, p)=1$ and $D t<\min \left(\ell-\xi_{0}+1, p^{e}\right)$.

Proof. This is a reformulation of Theorem 5.10 in type $\widetilde{A}_{1}$.

For a prime $p$, we denote by $\ell \ell(p)$ the lowest level of weight $\lambda$ such that $V(\lambda)$ is a reducible Weyl module arising from our main theorem. Note that there could be reducible Weyl modules that cannot be detected by our main Theorem with level lower than $\ell \ell(p)$. Table 2 below describes the first few $\ell \ell(p)$ and the possible high weights $\lambda=\left(\xi_{0}, \ell \ell(p)-\xi_{0}\right)$ of reducible Weyl modules detected by Corollary 6.7. One can observe that $\ell \ell(p)$ grows much slower than $p$.

TABle 2. The lowest level $\ell \ell(p)$ and possible $\xi_{0}$.

\begin{tabular}{c|cccccccc}
$p$ & 2 & 3 & 5 & 7 & 11 & 13 & 17 & 19 \\
\hline$\ell \ell(p)$ & 2 & 2 & 4 & 3 & 4 & 3 & 5 & 5 \\
\hline possible $\xi_{0}$ & $\{0,2\}$ & $\{0,2\}$ & $\{0,1,3,4\}$ & $\{1,2\}$ & $\{0,4\}$ & $\{0,3\}$ & $\{2,3\}$ & $\{0,5\}$ \\
$p$ & 23 & 29 & 31 & 37 & 41 & 43 & 47 & 53 \\
\hline$\ell \ell(p)$ & 5 & 6 & 7 & 6 & 7 & 7 & 7 & 8 \\
\hline possible $\xi_{0}$ & $\{2,3\}$ & $\{1,5\}$ & $\{3,4\}$ & $\{1,5\}$ & $\{2,5\}$ & $\{0,7\}$ & $\{2,5\}$ & $\{3,5\}$
\end{tabular}

The quasi-simple weights for $p=2,3$, and 5 up to level 150 are given in Table 3 below. In other words, any weight in $Y^{+}$that is not in the table corresponds to a reducible Weyl module. This supports the hypothesis that almost every Weyl module is reducible.

6.5. Type $\widetilde{A}_{r}: r \geq 2$. Assume that $\gamma=\alpha_{0}+t \delta$ with $t \geq 0$. Let $\left(\xi_{0}, \xi_{1}, \ldots, \xi_{r}\right)$ be the shorthand notation of the weight $\sum_{i=0}^{r} \xi_{i} \varpi_{i}$. Our main theorem leads to the following corollary.

Corollary 6.8. For type $\widetilde{A}_{r}, r \geq 2$ and a fixed prime $p$, a weight $\lambda=\left(\xi_{0}, \xi_{1}, \ldots\right.$, $\left.\xi_{r-1}, \ell-\left(\xi_{0}+\ldots+\xi_{r-1}\right)\right)$ of level $\ell$ lies in $Y^{+}$if and only if there are integers $M, D, e \in \mathbb{Z}_{>0}$ and $t \in \mathbb{Z}_{\geq 0}$ satisfying the following conditions.

(1) $\left(\ell+h^{\vee}\right) t+\xi_{0}+1=M p^{e}+D$. 
TABLE 3. Quasi-simple weights of level $<150$ in type $\widetilde{A}_{1}$.

\begin{tabular}{|c|c|c|c|c|c|}
\hline \multicolumn{2}{|r|}{$p=2$} & \multicolumn{2}{|r|}{$p=3$} & \multicolumn{2}{|r|}{$p=5$} \\
\hline$\ell$ & $\left(\xi_{0}, \ell-\xi_{0}\right)$ & $\ell$ & $\left(\xi_{0}, \ell-\xi_{0}\right)$ & $\ell$ & $\left(\xi_{0}, \ell-\xi_{0}\right)$ \\
\hline 3 & $(0,3),(3,0)$ & 3 & $(1,2),(2,1)$ & 2 & $(0,2),(2,0)$ \\
\hline 8 & $(1,7),(7,1)$ & 6 & $(1,5),(5,1)$ & 4 & $(2,2)$ \\
\hline 18 & $(3,15),(15,3)$ & 13 & $(5,8),(8,5)$ & 6 & $(3,3)$ \\
\hline 38 & $(7,31),(31,7)$ & 22 & $(5,17),(17,5)$ & 18 & $(4,14),(14,4)$ \\
\hline 78 & $(15,63),(63,15)$ & 43 & $(17,26),(26,17)$ & 28 & $(14,14)$ \\
\hline & & 70 & $(17,53),(53,17)$ & 38 & $(19,19)$ \\
\hline & & & & $\begin{array}{c}98 \\
148\end{array}$ & $\begin{array}{l}(24,74),(74,24) \\
(74,74)\end{array}$ \\
\hline
\end{tabular}

(2) $2 D \leq \xi_{0} \leq \ell-\left(\xi_{1}+\ldots+\xi_{r-1}\right)$.

In particular, if $\lambda \in Y^{+}$, the corresponding Weyl module $V(\lambda)$ is reducible if either Condition (i), (ii) or (iii) below holds:

(i) $\operatorname{gcd}(t+1, p)=1$ and $D(t+1)<\min \left(\xi_{0}+1, p^{e}\right)$.

(ii) $\operatorname{gcd}(t, p)=1$ and $D t<\min \left(\xi_{i}+1, p^{e}\right)$ for some $i=1, \ldots, r-1$.

(iii) $\operatorname{gcd}(t, p)=1$ and $D t<\min \left(\ell-\left(\xi_{0}+\ldots+\xi_{r-1}\right)+1, p^{e}\right)$.

Remark 6.9. We observe that, for a fixed type, every weight of level $\ell$ seems to be quasi-simple if $p \gg \ell$. In particular, for type $\widetilde{A}_{r}$ every weight in $Y^{+}$of level $\ell$ is quasi-simple if $p>\ell\left(\ell+h^{\vee}\right)-h^{\vee}$.

\section{REFERENCES}

[And80] H. H. Andersen, The strong linkage principle, J. Reine Angew. Math. 315 (1980), 53-59.

[BK02] J. Brundan and A. Kleshchev, Cartan determinants and Shapovalov forms, Math. Ann. 324 (2002), 431-449.

[Car05] R. W. Carter, Lie algebras of finite and affine type, Cambridge Studies in Advanced Mathematics 96, Cambridge University Press, Cambridge, 2005.

[CJ01] V. Chari and N. Jing, Realization of level one representations of $U_{q}(\hat{\mathfrak{g}})$ at a root of unity, Duke Math. J. 108 (2001), 183-197.

[CL74] R. W. Carter and G. Lusztig, On the modular representations of the general linear and symmetric groups, Math. Z. 136 (1974), 193-242.

[CP80] R. W. Carter and M. T. J. Payne, On homomorphisms between Weyl modules and Specht modules, Math. Proc. Cambridge Philos. Soc. 87 (1980), 419-425.

[DKK89] C. DeConcini, V. G. Kac, and D. A. Kazhdan, Boson-Fermion correspondence over Z, Infinite-dimensional Lie algebras and groups (Luminy-Marseille, 1988), Adv. Ser. Math. Phys. 7, World Sci. Publ., Teaneck, NJ, 1989, pp. 124-137.

[Don80] S. Donkin, The blocks of a semisimple algebraic group, J. Algebra 67 (1980), 36-53.

[Dot89] S. Doty, The strong linkage principle, Amer. J. Math. 111 (1989), 135-141.

[Fra81] J. Franklin, Homomorphisms between Verma modules and Weyl modules in characteristic $p$, Ph.D. thesis, University of Warwick, 1981. 
[Fra88] _ Homomorphisms between Verma modules in characteristic p, J. Algebra 112 (1988), 58-85.

[Gar78] H. Garland, The arithmetic theory of loop algebras, J. Algebra 53 (1978), 480-551.

[Hum71] J. E. Humphreys, Modular representations of classical Lie algebras and semisimple groups, J. Algebra 19 (1971), 51-79.

[Hum08] - Representations of semisimple Lie algebras in the BGG category $\mathcal{O}$, Graduate Studies in Mathematics 94, American Mathematical Society, Providence, RI, 2008.

[Jan77] J. C. Jantzen, Darstellungen halbeinfacher Gruppen und kontravariante Formen, J. Reine Angew. Math. 290 (1977), 117-141.

[Jan03] Representations of algebraic groups, second ed., Mathematical Surveys and Monographs 107, American Mathematical Society, Providence, RI, 2003.

[Kac90] V. G. Kac, Infinite-dimensional Lie algebras, third ed., Cambridge University Press, Cambridge, 1990.

[KK79] V. G. Kac and D. A. Kazhdan, Structure of representations with highest weight of infinite-dimensional Lie algebras, Adv. in Math. 34 (1979), 97-108.

[Kum90] S. Kumar, Bernstein-Gelfand-Gelfand resolution for arbitrary Kac-Moody algebras, Math. Ann. 286 (1990), 709-729.

[Mat96] O. Mathieu, On some modular representations of affine Kac-Moody algebras at the critical level, Compositio Math. 102 (1996), 305-312.

[Mit85] D. Mitzman, Integral bases for affine Lie algebras and their universal enveloping algebras, Contemporary Mathematics 40, American Mathematical Society, Providence, RI, 1985.

[Sha72] N. N. Shapovalov, On a bilinear form on the universal enveloping algebra of a complex semisimple lie algebra, Funct. Anal. Appl. 6 (1972), 307-312.

Department of Mathematics, University of Virginia, Charlottesville, VA 22904

E-mail address: cl8ah@virginia.edu 